\title{
Q.
QNEEN'S
UNIVERSITY
BELFAST
}

\section{Energy-Efficient and Throughput Fair Resource Allocation for TS- NOMA UAV-Assisted Communications}

\author{
Masaracchia, A., Nguyen, L. D., Duong, T. Q., Yin, C., Dobre, O. A., \& Garcia-Palacios, E. (2020). Energy- \\ Efficient and Throughput Fair Resource Allocation for TS-NOMA UAV-Assisted Communications. IEEE \\ Transactions on Communications. https://doi.org/10.1109/TCOMM.2020.3014939
}

\section{Published in:}

IEEE Transactions on Communications

\section{Document Version:}

Peer reviewed version

\section{Queen's University Belfast - Research Portal:}

Link to publication record in Queen's University Belfast Research Portal

\section{Publisher rights}

(C) 2020 IEEE.

This work is made available online in accordance with the publisher's policies. Please refer to any applicable terms of use of the publisher.

\section{General rights}

Copyright for the publications made accessible via the Queen's University Belfast Research Portal is retained by the author(s) and / or other copyright owners and it is a condition of accessing these publications that users recognise and abide by the legal requirements associated with these rights.

Take down policy

The Research Portal is Queen's institutional repository that provides access to Queen's research output. Every effort has been made to ensure that content in the Research Portal does not infringe any person's rights, or applicable UK laws. If you discover content in the Research Portal that you believe breaches copyright or violates any law, please contact openaccess@qub.ac.uk. 


\title{
Energy-Efficient and Throughput Fair Resource Allocation for TS-NOMA UAV-Assisted Communications
}

\author{
Antonino Masaracchia, Long D. Nguyen, Trung Q. Duong, Cheng Yin, Octavia A. Dobre and Emiliano \\ Garcia-Palacios
}

\begin{abstract}
This paper proposes an optimization framework for power and time resource allocation during time sharing nonorthogonal multiple access (TS-NOMA) transmissions performed by an unmanned aerial vehicle (UAV) in the context of a largescale scenario. The objective of the proposed UAV-TS-NOMA system and optimization framework is to jointly maximize the energy efficiency (EE) and the downlink throughput fairness among users within the UAV communication range. The idea behind is to propose a communication system that: $i$ ) merges the advantages of UAV communications with the ones offered by the TS-NOMA paradigm and ii) maximizes the EE and the downlink fairness among users. The resulting model finds applicability in performing energy efficient and throughput fair transmissions into power-constrained communication scenarios. Performance investigations regarding the proposed framework in finding the optimal set of resources which maximizes jointly the above mentioned network metrics, have shown the advantage of the proposed two-step optimization framework in finding the optimal configuration of both power and time resources, respecting both the power constraints at the transmitter and the quality-of-service requirement of the users. In addition, it is shown how under particular conditions the proposed framework jointly optimizes the aforementioned network metrics in only one step.
\end{abstract}

Index Terms-EE, NOMA, throughput fairness, TS-NOMA, UAV communications..

\section{INTRODUCTION}

According to Cisco forecast [1], in the near future seven trillion wireless devices will serve seven billion people, generating a huge amount of data traffic, i.e., around 50 exabytes of monthly global mobile data traffic. Under this perspective, $5 \mathrm{G}$ networks are being standardized to provide wide coverage, support more devices, and achieve higher throughput. To meet

This work was supported in part by the U.K. Royal Academy of Engineering Research Fellowship under Grant RF1415 \14\22 and by a Researcher Links grant, ID 528154944, under the Newton Fund partnership. The grant is funded by the UK Department for Business, Energy and Industrial Strategy and delivered by the British Council, for further information, please visit www.newtonfund.ac.uk. The work of O. A. Dobre was supported in part by The Natural Sciences and Engineering Research Council of Canada (NSERC) through its Discovery program.

Antonino Masaracchia, Cheng Yin, T. Q. Duong, and E. Garcia-Palacios are with the School of Electronics, Electrical Engineering and Computer Science, Queens University Belfast, Belfast BT7 1NN, U.K. (e-mail: A.Masaracchia@qub.ac.uk; cyin01@qub.ac.uk; trung.q.duong@qub.ac.uk; e.garcia@ee.qub.ac.uk).

Long D. Nguyen is with the Duy Tan University, Da Nang city, 550000, Vietnam (e-mail: dinhlonghcmut@gmail.com).

O. A. Dobre is with Faculty of Engineering and Applied Science, Memorial University, Canada (e-mail: odobre@mun.ca).

Corresponding author is T. Q. Duong. these overwhelming requirements, the design of a suitable radio access technology (RAT) represents an important aspect for improving the capacity of a cellular mobile communication system in a cost-effective manner.

Non-orthogonal multiple access (NOMA) technology offers a number of advantages which permit to label it as a promising multiple access scheme for future RAT [2][4], gaining a great attention from both academia research and industry. The basic idea of NOMA is to serve multiple users in the same resource (time/frequency/code) block (RB). A way to make this is through power-domain superposition coding (SC) multiplexing at the transmitter and successive interference cancellation (SIC) at the receiver [5]. On the other hand, unmanned aerial vehicle (UAV) communication is an emerging technique which, due to its mobility, flexibility and good channel condition, can help future networks to achieve better performances [6], [7]. Indeed, in contrast to the conventional static base station (BS) communication, a distinct feature of the UAV communication is that the existence of line-of-sight (LoS) is capable of offering reduced smallscale fading between UAVs and ground users, providing the possibility to increase network performances. In other words, the main distinctive feature between UAV communication and an elevated BS is represented by the fact that the UAV position can be dynamically optimized, permitting to improve channel gains statistics of users and then network metric performances [8]. For this reason, recently the usage of UAV communication has been also emerging as a paradigm in the context of disaster relief networks, allowing to optimize both UAV's position and trajectory in order to provide as much coverage as possible to the users within the disaster area and supporting a timely response of first aid operations [9]-[11].

The possibility to employ NOMA for future UAV communication systems has been recently recognized as a very effective solution to reach the massive spectral efficiency requirements for the 5G/Beyond-5G wireless networks [12]. Under this perspective, several research studies have been conducted in order to analyze the critical aspects of this possible communication architecture. Dynamic power allocation strategies, jointly optimized with the UAV altitude, have been proposed in [13]-[15]. In particular, supposing that only two users are multiplexed in power domain, power allocation strategies have been proposed in order to improve user-access fairness [13], throughput [14], as well as coverage [15] in UAV-enabled enabled communication using NOMA. However, all these studies 
supposed that only two users are multiplexed in power domain. Power allocation methodologies for maximizing throughput in a quasi-static NOMA UAV-BS deployment have been presented in [16]. Although in this work it is assumed that more than two users can be multiplexed in power domain, the joint optimization of UAV altitude and performance dynamics resulting from the choice of user pairing schemes was not discussed. A user scheduling algorithm for a fixed altitude cyclical NOMA UAV communication system has been detailed in [17]. In this study, multiple users are multiplexed in power domain in a cyclical manner. Although this type of approach can result in a more fair resource allocation in terms of user perceived throughput, the assumed simplification of predetermined power allocation while ignoring individual qualityof-service (QoS) requirements may lead to unfair resource allocation between the paired users. A joint optimal power allocation and placement for NOMA-UAV networks has been presented in [18]. The location of the UAV is first optimized and used to determine the power allocation for NOMA which maximizes the sum rate of the network. In this case the QoS requirements of each user are included in the problem formulation.

Despite the high quality research works recently presented in literature, the performance of an UAV assisted communication system is often limited by the power constraint on the on-board energy reserves. Then, the energy efficiency (EE), defined as the ratio of downlink sum-rate to the total power consumption, has emerged as a core performance metric for the UAV based wireless networks [19]. An energy efficient placement algorithm for a drone BS that serves a set of ground users, using minimum required transmit power was proposed in [20], [21]. In both works, the optimal drone position is obtained by decoupling the deployment problem in the horizontal and vertical dimensions. With respect to [20], authors in [21] illustrated how the performance of the proposed placement framework in terms of power saving varies within the user distribution. Finally, iterative trajectory planning strategies aiming to minimize the total energy consumption and maximize the secrecy energy efficiency of a fixed-wing UAV-BS have been proposed in [22] and [23], respectively. However, the reported analyses assumed that orthogonal multiple access (OMA) techniques are employed. In addition to the EE maximization problem investigated in the context of NOMA-enabled static BS networks in both transmission directions [24], [25], i.e., downlink and uplink, recently, studies have focused on the EE maximization problem for UAV-enabled networks in which NOMA technology is adopted [26], [27]. In particular, authors in [26] proposed a two-sided matching and swapping algorithm to achieve subchannel assignment and a power allocation to iteratively maximize the EE of a fog UAV wireless network. Through simulation they have shown how the EE of NOMA-based fog UAV wireless networks is higher than that of an orthogonal frequency-division multiple access scheme. A more exhaustive investigation where the UAV altitude and the QoS constraint of the users are considered in optimization problem formulation was conducted in [27].

In the context of NOMA user aggregation procedure, the most common approach, adopted in the aforementioned works, is to multiplex a cell centre user (best channel gain) with a cell edge user (worse channel gain). This user pairing strategy is sometimes termed as conventional NOMA (C-NOMA). However, due to the random distribution of users, the ratio of users in near and far regions may vary significantly, especially in the case of UAV communications where the channel gain conditions are dependent on the UAV altitude [6], [7]. In order to address the case in which the number of cell centre users is less than cell edge users, a virtual pairing based NOMA (VP-NOMA) was proposed in [28]. The VP-NOMA system simultaneously pairs a cell centre user with two or more cell edge users over non-overlapping frequency bands, achieving higher capacity gains compared to C-NOMA and OMA techniques. However, the cell centre user may need to perform multiple SIC operations, increasing the power consumption of the decoding users, which in some power constrained scenarios such as disaster communications is a critical issue [29], [30]. In order to reduce this computational cost, a time sharing-NOMA (TS-NOMA) system was proposed in [31]. This approach consists in dividing the total time slot duration $T$ into two sub-time slots $t_{1}$ and $t_{2}$. During the first sub-time slot $t_{1} \leq T, \mathrm{UE}_{1}$ (cell centre user) is paired with $\mathrm{UE}_{2}$ (cell edge user) . For the remaining time $t_{2}=T-t_{1}, \mathrm{UE}_{1}$ is paired with another cell edge user, i.e., $\mathrm{UE}_{3}$. In this way, $\mathrm{UE}_{1}$ is able to use the whole bandwidth of $B \mathrm{~Hz}$ for the complete duration of communication session, whereas $\mathrm{UE}_{2}$ and $\mathrm{UE}_{3}$ can use the complete bandwidth for portions of time $t_{1}$ and $t_{2}$, respectively. In addition, the computational cost at the cell centre user is reduced since only one SIC process per sub-time slot is performed.

As far as the authors are aware, the technical literature lacks works related to the application of the TS-NOMA concept to UAV-NOMA communications (UAV-TS-NOMA), especially in the context of disaster relief network where this communication paradigm can be adopted as a potential solution to perform energy-efficient and throughput fair transmissions by saving energy at both transmitter and receiver. Thus, this paper aims to fill in the existing gap in the literature. Specifically, the main contributions of this paper are as follow:

- We propose a model of an UAV-TS-NOMA system and for such a communication model, in addition to a low computational cost user clustering procedure, we formulate a joint $\mathrm{EE}$ and throughput fairness optimization problem which takes into account the QoS requirements of users and the power availability at the UAV as optimization constraints. To the best of the authors' knowledge, this represents the first work on joint EE and throughput fairness maximization for a TS-NOMA applied in the context of a large-scale network scenario under UAV-enabled communication.

- Due to the non-linearity and non-convexity of the EE optimization problem, we propose a dual-layer iterative algorithm for the optimal resource allocation, which basically finds the joint sub-optimal resource allocation scheme in two steps. The first step aims to select the proper power resources to maximize the EE. Then, the 
second optimization step aims to select the proper time resources to maximize the throughput fairness among users. More importantly, we propose semi-closed form solutions for both steps. In addition, under particular conditions, the power optimization step is sufficient to jointly optimize the aforementioned network metrics, reducing the processing energy consumed by the UAV for evaluating the optimal set of power resources.

- We conduct a performance investigation campaign through simulations varying the main parameters that influence the optimization process, and showing how the proposed framework is able to find the optimal resource allocation configuration while satisfying the constraints. In particular, we show how the proposed communication paradigm achieves higher network performances with a lower computational complexity when compared with another EE framework proposed in the literature.

The rest of the paper is organized as follows. The system model, as well as a user aggregation policy and the formulation of the joint power and time resource allocation optimization problem are introduced in Section II. Section III provides the proposed two-step procedure for joint power and time resource allocation. Section IV presents the performance investigation of the proposed approach along with a comparison with a random power allocation scheme, a fixed power allocation schemes and another EE power allocation scheme proposed in literature. Finally, conclusions and future directions are drawn in Section V.

\section{System Model And Problem Formulation}

As illustrated in Fig. 1, let us suppose to have a set of $N$ users, randomly distributed into a circular area of radius $R$ according to a Poisson point process (PPP) and served by an UAV which performs TS-NOMA transmissions. Each time slot $T$ is divided in $N_{S}=2$ time slots, i.e. $t_{1}=\alpha T$ and $t_{2}=(1-\alpha) T$, with $0 \leq \alpha \leq 1$. The UAV is placed at the center of the cell at height $H$. To perform energy efficient TS-NOMA transmissions among its users, the UAV should perform the following operations: $i$ ) classify the users according to their channel condition obtaining Type-I (cell centre) and Type-II (cell edge) users, ii) adopt a clustering policy in order to multiplex Type-I users with Type-II users in the power domain, and iii) select both the optimal power coefficients and the time slot durations in order to jointly maximize the EE and the downlink throughput fairness among users.

\section{A. Node classification and clustering}

The full procedure for node classification and clustering is summarized in Algorithm 1 and described in the sequel. Supposing that the UAV has complete knowledge about the channel gain of each user, a very fast and simple way to divide the set of users into two clusters is represented by the adoption of the $k$-means ++ algorithm [32]. The output of this algorithm consists in a group of $N_{C}$ cell centre users and a group of $N_{E}$ cell edge users. Once the two subsets of users are obtained, iteratively each cell centre user is paired with

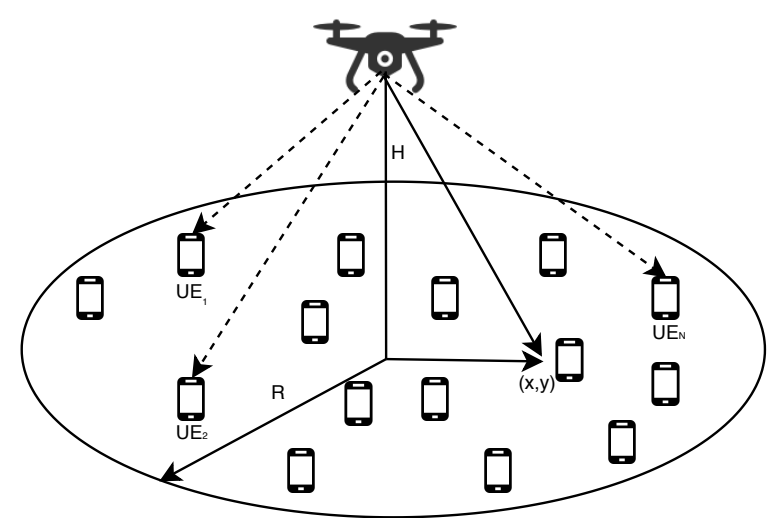

Fig. 1. System model.

$K$ cell edge users per sub-time slot, i.e., $2 K$ cell edge users in total. Under this assumptions, the ideal output from the $k$ means algorithm should consist in $\frac{N}{2 K+1}$ cell centre users and $N \times \frac{2 K}{2 K+1}$ edge cell users. However the output is dependent on the channel gain conditions. Then, when $N_{C}<\frac{N}{2 K+1}$ we modify the $k$-means ++ algorithm in order to consider the first $K_{1}=\left(\frac{N}{2 K+1}-N_{C}\right)$ strong users from the $N_{E}$ as cell centre users. The opposite procedure is applied when $N_{C}>\frac{N}{2 K+1}$, i.e., the last $K_{2}=N_{C}-\frac{N}{2 K+1}$ are considered as weak users. ${ }^{1}$ In Fig. 2 one can observe the system model after node classification, where the green area and red area contains cell centre and cell edge users, respectively. Once these clusters are obtained, the available bandwidth is divided into $N_{C}$ orthogonal sub-channels labelled with an unique ID, i.e., $I D=1, \cdots, N_{C}$, each of them used to multiplex a cell centre user with the corresponding $2 K$ cell edge users in the power domain. Since the user pairing process and user to sub-channel mapping represents a critical aspect for NOMA systems [33], [34], indicating with $g_{i, c}$ the channel gain of the $i$-th cell centre user and with $g_{k, e}$ the channel gain of the $k$-th cell edge user, they will be multiplexed in the power domain if the channel gain ratio $\frac{g_{k, e}}{g_{i, c}}$ fits in the range reported in Table I, which guarantees the minimum power requirement at the transmitter [35].

TABLE I

ChanNEL Gain RATIOS.

\begin{tabular}{|c|c|c|c|}
\hline Edge users per time slot & $K=1$ & $K=2$ & $K=3$ \\
\hline \multirow{3}{*}{ channel gain ratios } & {$[0.4-0.5]$} & {$[0.4-0.5]$} & {$[0.4 ; 0.5]$} \\
\cline { 2 - 4 } & & {$[0.2-0.35]$} & {$[0.2-0.35]$} \\
\cline { 3 - 4 } & & & {$[0.5-0.15]$} \\
\hline
\end{tabular}

\section{B. Resource allocation problem}

As mentioned before, we suppose that the available bandwidth $B$ is divided into $N_{C}$ orthogonal sub-channels of equal size $B / N_{C}$. Each sub-channel is used to multiplex one Type-I user with $K_{i, 1}$ and $K_{i, 2}$ Type-II users during the first and the

\footnotetext{
${ }^{1}$ These assumptions have been considered in order to obtain a complete performance investigation varying the UAV altitude, which impacts on channel conditions.
} 


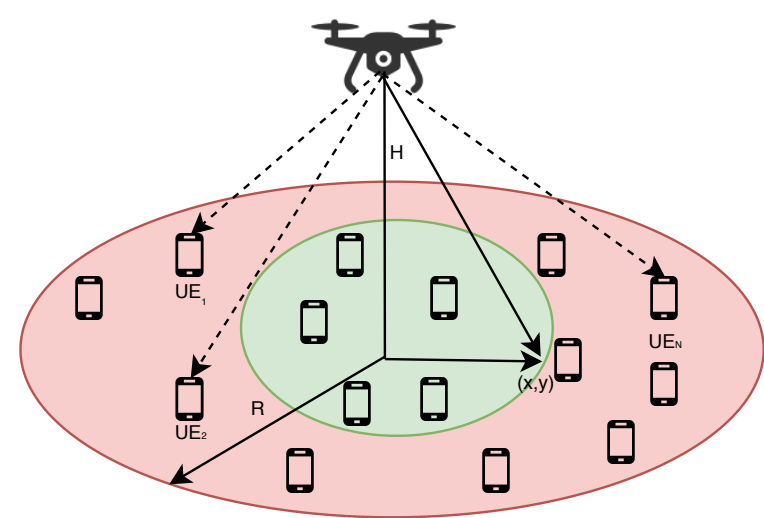

Fig. 2. System model after node classification.

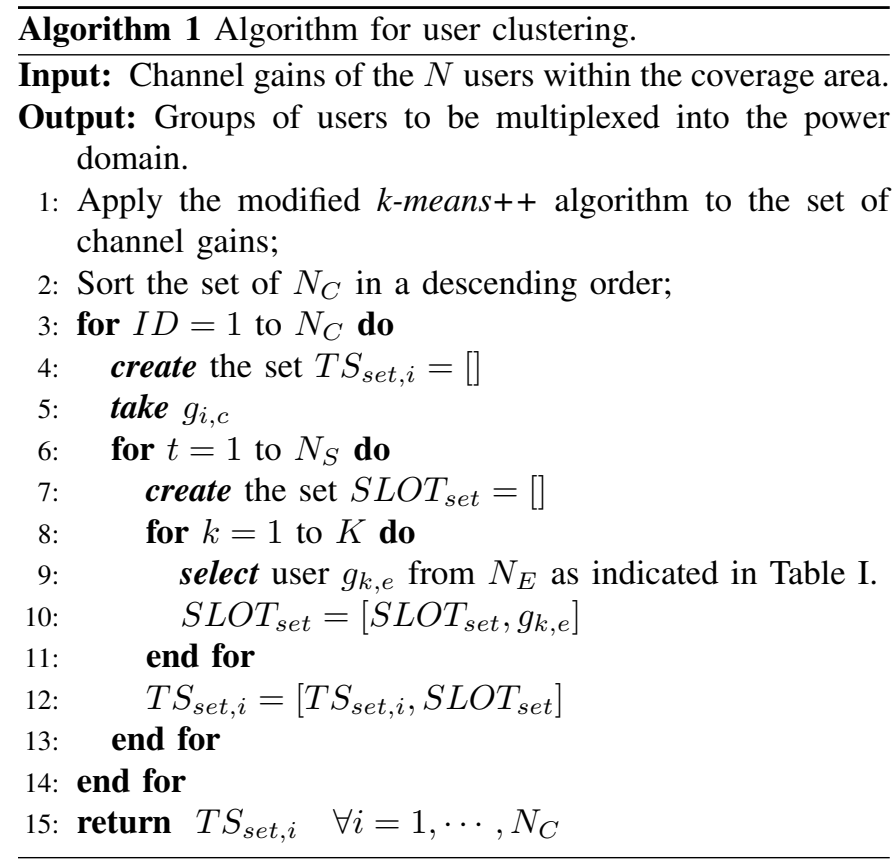

second sub-time slot, respectively. Then, the signal received by the generic $k$-th user within the $i$-th sub-channel during the first sub-time slot, can be expressed as:

$$
y_{i, k, 1}=h_{k} \times \sum_{j=1}^{K_{i, 1}+1} \sqrt{P_{j}} s_{j}+\omega_{k},
$$

where $h_{k}$ represents the channel coefficient of user $k, P_{j}$ is the amount of transmitting power allocated to user $j, s_{j}$ with $\left\|s_{j}\right\|^{2}=1$, is the signal transmitted to user $j$ and $\omega_{k}$ is the received noise.

Regarding the channel coefficient $h_{k}$, it has been modelled using the air-to-ground (ATG) channel model for device-todevice UAV-assisted communication provided in [36], [37]. In particular, the channel coefficient from the UAV to the $k$-th user located at $(x, y)$ position can be written as:

$$
\begin{aligned}
h_{k}= & P_{r L O S} \times\left(\sqrt{x^{2}+y^{2}+H^{2}}\right)^{-\beta} \\
& +P_{r N L O S} \times \gamma\left(\sqrt{x^{2}+y^{2}+H^{2}}\right)^{-\beta},
\end{aligned}
$$

where $\beta$ represents the path-loss exponent and $\gamma$ is the excessive attenuation factor in the case of non-LoS (NLoS). Finally, $P_{r L O S}$ and $P_{r N L O S}=1-P_{r L O S}$ represent the LoS and the NLoS probability, respectively. These last two quantities are obtained from the following formula [36]:

$$
P_{r L O S}=\frac{1}{1+a \times \exp (-b[\phi-a])},
$$

where $a$ and $b$ are constant values depending on the environment, and $\phi$ is the elevation angle between the UAV and the user expressed in degree, as follow:

$$
\phi=\frac{180}{\pi} \times \arcsin \left(\frac{H}{\sqrt{x^{2}+y^{2}+H^{2}}}\right) .
$$

Furthermore, due to the random distribution of obstacles between UAV and UEs, for a more realistic mobile communication model [38], random components $\zeta_{L O S}$ and $\zeta_{N L O S}$ following a log-normal distribution with a zero mean and standard deviations $\sigma_{L O S}$ and $\sigma_{N L O S}$ are considered, respectively. Then, (2) can be expressed as:

$$
\begin{aligned}
h_{k}= & P_{r L O S} \times \zeta_{L O S}\left(\sqrt{x^{2}+y^{2}+H^{2}}\right)^{-\beta} \\
& +P_{r N L O S} \times \zeta_{L O S} \times \gamma\left(\sqrt{x^{2}+y^{2}+H^{2}}\right)^{-\beta}
\end{aligned}
$$

In the same way, the signal received by the generic $k-t h$ user during the second sub-time slot is:

$$
y_{i, k, 2}=h_{k} \times \sum_{j=1}^{K_{i, 2}+1} \sqrt{P_{j}} s_{j}+\omega_{k}
$$

Then, according with (1) and (6), the achievable rate of each user during the respective time slot can be expressed as:

$$
R_{i, k, 1}=\alpha \frac{B}{N_{C}} \log _{2}\left(1+\frac{\left|h_{k}\right|^{2} P_{k}}{\sigma^{2}+\left|h_{k}\right|^{2} \sum_{j=k+1}^{K_{i, 1}+1} P_{j}}\right),
$$

and

$$
R_{i, k, 2}=(1-\alpha) \frac{B}{N_{C}} \log _{2}\left(1+\frac{\left|h_{k}\right|^{2} P_{k}}{\sigma^{2}+\left|h_{k}\right|^{2} \sum_{j=k+1}^{K_{i, 2}+1} P_{j}}\right)
$$

Then, the total amount of power employed by the UAV is represented by:

$$
P_{\text {total }}=\zeta\left(\alpha \sum_{j=1}^{K_{i, 1}+1} P_{j}+(1-\alpha) \sum_{j=1}^{K_{i, 2}+1} P_{j}\right)+P_{\text {circ }}
$$

where $\zeta$ is the reciprocal of the drain efficiency of the amplifier and $P_{\text {circ }}$ is the non-transmissive power dissipated into the internal power circuit of the transmitter. Indicating with $\mathbf{P}$ the vector containing all the power levels allocated for each user 
during the first and the second time slot, the EE optimization problem per sub-channel is formulated as follow:

$$
\begin{array}{ll}
\max _{\mathbf{P} ; \alpha} & \frac{\sum_{k=1}^{K_{i, 1}+1} R_{i, k, 1}+\sum_{k=1}^{K_{i, 2}+1} R_{i, k, 2}}{P_{\text {tot }}} \\
\text { s.t. } \quad & R_{i, k} \geq R_{i, k}^{\text {min }}, \quad \forall k=1 \cdots K_{\text {tot }, i} \\
& \sum_{k=1}^{K_{i, 1}} P_{k} \leq P_{\text {sub }} \\
& \sum_{k=1}^{K_{i, 2}} P_{k} \leq P_{\text {sub }} \\
& 0 \leq \alpha \leq 1,
\end{array}
$$

where $K_{t o t, i}=K_{i, 1}+K_{i, 2}+2$. Equation (10b) represents the QoS constraint of each user in downlink and (10c)-(10d) represent the constraints on the power transmission level. In particular, supposing that $P_{\max }$ is the maximum transmission power along the whole bandwidth, we assume that it is equally distributed within each sub-channel, i.e., $P_{s u b}=P_{\max } / N_{C}$.

\section{Joint Power And Time Resource Allocation AlgORITHM}

In this section we illustrate the proposed joint power and time resource allocation framework for an UAV-TS-NOMAenabled system. Due to the non-linearity of (10) we propose a dual-layer iterative algorithm, organized in two steps as follow:

1) Optimal power allocation procedure utilized to find the optimal vector $\mathbf{P}$ which maximizes the $\mathrm{EE}$ for a fixed value of $\alpha$;

2) Optimal time resource allocation procedure to update the factor $\alpha$, using the power allocation values $\mathbf{P}$ obtained from the previous step.

\section{A. Power allocation procedure}

As stated before, during the first optimization step the time factor $\alpha$ is considered as constant in the range $[0,1]$. Then, the optimization problem (10) can be considered as a power allocation problem for EE maximization as follows:

$$
\begin{array}{ll}
\max _{\mathbf{P}} & \frac{\sum_{k=1}^{K_{i, 1}+1} R_{i, k, 1}+\sum_{k=1}^{K_{i, 2}+1} R_{i, k, 2}}{P_{\text {tot }}} \\
\text { s.t. } \quad & R_{i, k} \geq R_{i, k}^{\text {min }}, \quad \forall k=1 \cdots K_{\text {tot }, i} \\
& \sum_{k=1}^{K_{\text {tot }, i}} P_{k} \leq P_{\text {sub }} .
\end{array}
$$

Since (11) can be classified as a non-linear fractional optimization problem, we propose to apply the Dinkelbach method [39], which as illustrated in [40], can be used for solving general non-linear fractional programming problems. In particular, according with the Dinkelbach method, the vector $\mathbf{P}^{*}$ solves the problem (11) if and only if it solves the following equivalent problem:

$$
\begin{aligned}
& \max _{\mathbf{P}} \quad\left(\sum_{k=1}^{K_{i, 1}+1} R_{i, k, 1}+\sum_{k=1}^{K_{i, 2}+1} R_{i, k, 2}\right)-\tau \times P_{t o t} \\
& \text { s.t. } \quad R_{i, k} \geq R_{i, k}^{\min }, \quad \forall k=1 \cdots K_{t o t, i} \\
& \quad \sum_{k=1}^{K_{t o t, i}} P_{k} \leq P_{\text {sub }},
\end{aligned}
$$

with $\tau=\left.\tau^{*} \triangleq \frac{\sum_{k=1}^{K_{i, 1}+1} R_{i, k, 1}+\sum_{k=1}^{K_{i, 2}+1} R_{i, k, 2}}{P_{\text {tot }}}\right|_{\mathbf{P}=\mathbf{P}^{*}} . \quad$ After some mathematical manipulations, the objective function can be expressed as:

$$
\alpha \times\left[\hat{R}_{i, 1}-\tau \hat{P}_{i, 1}\right]+(1-\alpha) \times\left[\hat{R}_{i, 2}-\tau \hat{P}_{i, 2}\right],
$$

where $\hat{R}_{i, 1}=\sum_{k=1}^{K_{i, 1}+1} \frac{B}{N_{C}} \log _{2}\left(1+\frac{g_{k} P_{k}}{\sigma^{2}+g_{k} \sum_{j=k+1}^{K_{i, 1}+1} P_{j}}\right)$ and $\hat{P}_{i, 1}=\zeta \sum_{k=1}^{K_{i, 1}+1} P_{k}+P_{\text {circ }}$. The values $\hat{P}_{i, 2}$ and $\hat{R}_{i, 2}$ are defined in a similar way. As final result, the original fractional problem (10) has been reduced to two optimization subproblems, one for each sub-time slot, which can be studied separately. Henceforth, since the two sub-problems are in the same form, without loss of generality we will refer to the first sub-time slot problem. Then, indicating with $\mathbf{P}_{\mathbf{1}}$ the variable of the first sub-problem which contains all the power levels used during the first sub-time slot, the new optimization problem becomes:

$$
\begin{aligned}
& \max _{\mathbf{P}_{1}}\left[\hat{R}_{i, 1}-\tau \hat{P}_{i, 1}\right] \\
& \text { s.t. } \quad R_{i, k} \geq R_{i, k}^{\text {min }}, \quad \forall k=1 \cdots K_{i, 1}+1 \\
& \sum_{k=1}^{K_{i, 1}+1} P_{k} \leq P_{\text {sub }} .
\end{aligned}
$$

However, due to the non-concavity form of the achievable rate function $\hat{R}_{i, 1}$, the problem (14) is still difficult to solve. To overcome this issue, we use efficient mathematical techniques to transform problem (14) into a convex form. Firstly, constraint (14b) can be relaxed by setting $\hat{P}_{k} \triangleq A_{k} / \phi_{k}$, where $A_{k}=\left(2^{\frac{R_{i, k}^{m i n} \times N_{C}}{\alpha \times B}}\right)$ and $\phi_{k}=g_{k} /\left(\sigma^{2}+g_{k} \sum_{j=k+1}^{K_{i, 1}+1} P_{j}\right)$. Therefore, the power variable can be rewritten as $P_{k}=\hat{P}_{k}+\tilde{P}$, where $\tilde{P}_{k} \geq 0$ and $\hat{P}_{k}$ represents the minimum amount of power necessary to maintain the QoS of each user. Then, (14) is equivalent to the following convex optimization problem

$$
\begin{aligned}
\max _{\tilde{\mathbf{P}}_{\mathbf{1}}} & \sum_{k=1}^{K_{i, 1}+1} \frac{B}{N_{C}} \log _{2}\left(a_{k}+\phi_{k} \tilde{P}_{k}\right)-\tau \hat{P}_{i, 1}(\tilde{\mathbf{P}}) \\
\text { s.t. } & \sum_{k=1}^{K_{i, 1}} \tilde{P}_{k} \leq \hat{P}_{\text {sub }},
\end{aligned}
$$

where $\hat{P}_{\text {sub }}=\alpha P_{\text {sub }}-\sum_{k=1}^{K_{i, 1}} \hat{P}_{k}, a_{k}=1+\phi_{k} \hat{P}_{k}$ and $P_{k}$ is defined as 


$$
P_{k}=\left\{\begin{array}{l}
\frac{A \sigma^{2}}{g_{k}}+\tilde{P}_{k} \quad k=1, \\
\frac{A \sigma^{2}}{g_{k}}+A \sum_{j=1}^{k-1} \hat{P}_{j}+A \sum_{j=1}^{k-1} \tilde{P}_{j}, \quad k>1 .
\end{array}\right.
$$

Remembering that $P_{k}=\hat{P}_{k}+\tilde{P}$, for a sake of simplicity and without loss of generality we can derive the following expressions:

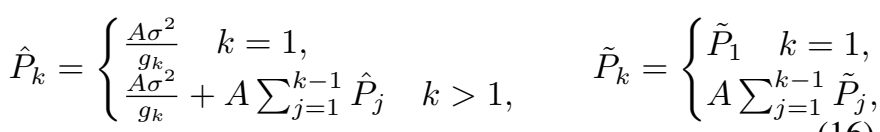

Then, once $\hat{P}_{1}$ and $\tilde{P}_{1}$ are calculated, the expressions in (16) can be used to calculate all the power levels recursively. However, as stated before, $\hat{P}_{k}$ represents the minimum amount of power necessary to maintain the QoS of each user. Then, in order to set the initial level of power of each user we employ the procedure described in the sequel. Supposing that $\tilde{P}_{1}$ has been randomly allocated, after some mathematical manipulations, the expression for the generic $\tilde{P}_{k}$ illustrated in (16) can be written as:

$$
\tilde{P}_{k}=\left\{\begin{array}{l}
\tilde{P}_{1} \quad k=1, \\
(1+A)^{k-2} A \times \tilde{P}_{1}, \quad k>1 .
\end{array}\right.
$$

Then, by applying the power constraint (15b), after some mathematical manipulation, we obtain:

$$
\sum_{k=1}^{K_{i, 1}} \tilde{P}_{k}=\tilde{P}_{1}+\sum_{k=2}^{K_{i, 1}}(1+A)^{k-2} A \times \tilde{P}_{1}=\tilde{P}_{1} \times(1+A)^{K_{i, 1}-1}
$$

In other words, we found that $\tilde{P}_{1} \leq \frac{\hat{P}_{s u b}}{(1+A)^{K_{i, 1}-1}}$. From this constraint, is possible to perform the random initialization of problem (15) as illustrated in Algorithm 2. When the

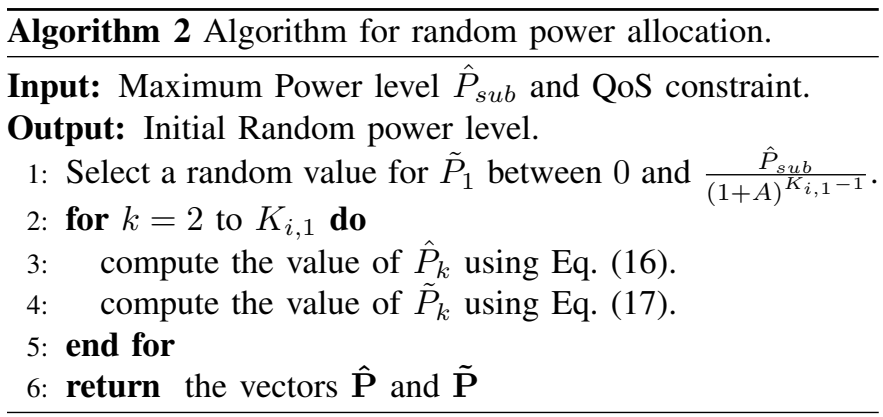

initialization process is completed, the problem (15) is solved through the following algorithm:

I ) Initialization. Solve (15a) for initial $\tau>0$. If its optimal value is higher than zero, set $\underline{\tau}=\tau$, reset $\tau \leftarrow 2 \tau$, and solve (15a) again. Otherwise (its optimal value is less than zero), set $\bar{\tau}=\tau$. We end up having $\underline{\tau}$ and $\bar{\tau}$ such that the optimal value of (15a) is positive for $\tau=\underline{\tau}$ and negative for $\tau=\bar{\tau}$. The optimal $\tau$ for zero optimal value of (15a) lies on $[\underline{\tau}, \bar{\tau}]$; as such, we locate it by the bisection method, as explained in the following.

II ) Bisection. Solve (15a) for $\tau=(\underline{\tau}+\bar{\tau}) / 2$. If its optimal value is positive, reset $\underline{\tau} \leftarrow \tau$, otherwise (its optimal value is negative), reset $\bar{\tau} \leftarrow \tau$. This process is repeated until the condition $\bar{\tau}-\underline{\tau} \leq \epsilon$ (tolerance) is reached. Then, optimal value of (15a) will be $\tau^{*}=(\underline{\tau}+\bar{\tau}) / 2$.

III ) Optimal power $\tilde{P}_{k}^{*}$ computation. For a fixed value $\tau^{*}$ obtained from the previous step, compute the gradient of (15a) respect to the $\mathbf{P}$ variable. Then, the optimal value $\mathbf{P}^{*}$ is the one at which the gradient is equal to zero.

From the previous procedure, starting to compute the firstorder derivative from the user with worst channel condition and defining the $f(\tilde{\mathbf{P}})=\sum_{k=1}^{\bar{K}} \frac{B}{N_{C}} \log _{2}\left(a_{k}+\phi_{k} \tilde{P}_{k}\right)-$ ${ }_{T}^{k} \vec{P}_{i, 1}{ }^{1}(\tilde{\mathbf{P}})$ and $\bar{K}=K_{i, 1}+1$, the execution of step III consists in finding the solution of the following system of $\bar{K}$ equations:

$$
\nabla f(\tilde{\mathbf{P}})=\left\{\begin{array}{l}
\frac{B}{N_{C}} \times \frac{\phi_{\bar{K}}}{\Psi\left(\bar{P}_{\bar{K}}\right)}-\tau \zeta \\
\vdots \\
\frac{B}{N_{C}} \times\left(\frac{\phi_{k}}{\Psi\left(\tilde{P}_{k}\right)}+\sum_{j=k+1}^{\bar{K}} \frac{\phi_{j}^{\prime}}{\Psi\left(\tilde{P}_{j}\right)}\right)-\tau \zeta
\end{array}=\mathbf{0}\right.
$$

where $\Psi\left(P_{k}\right)=\ln (2)\left(a_{k}+\phi_{k} \tilde{P}_{k}\right)$ and $\phi_{j}^{\prime}=\frac{\partial \phi_{j}}{\partial \tilde{P}_{j}}$. Even if this provides a closed-form expression for the optimal solution, as the number of users increases, it can result in high computational cost procedure, requiring high levels of energy for the computation of the solution. Under this perspective, we propose a sub-optimal approach for the computation of power coefficients. In particular, solving the first equation of the previous system, $P_{\bar{K}}$ has the following closed-form expression:

$$
\leq \hat{P}_{\text {sub }} . \quad \tilde{P}_{\bar{K}}=\frac{B / N_{C}}{\ln (2) \tau \zeta}-\frac{a_{\bar{K}}}{\phi_{\bar{K}}} .
$$

At this step, once the optimal power level of the user with worst channel condition has been obtained, we can calculate the other coefficients exploiting the relation in (16). In particular, if $\bar{K}=2$ :

$$
0 \leq \tilde{P}_{1} \leq \frac{\tilde{P}_{\bar{K}}}{A} .
$$

Otherwise, if $\bar{K}>2$

$$
0 \leq \tilde{P}_{k} \leq \begin{cases}\frac{\tilde{P}_{\bar{K}}}{(1+A)^{K-k}}, & 2 \leq k \leq \bar{K}-1 \\ \frac{P_{\bar{K}}}{(1+A)^{K-k} A}, & k=1\end{cases}
$$

According to (21) and (22), considering users as decreasing ordered pairs, i.e., $\left(P_{\bar{K}} ; P_{\bar{K}-1}\right),\left(P_{\bar{K}-1} ; P_{\bar{K}-2}\right), \cdots,\left(P_{2} ; P_{1}\right)$, one can notice that for each of these pairs the power for the user with best channel condition should be considered as a fraction of the power of user with worst channel condition. Indicating with $\beta_{P}$ this fraction which respects conditions (21)-(22), as reported in Fig. 3a, one can observe how an increase of $\beta_{P}$ corresponds a decrease/increase of downlink user perceived throughput of user with worst/better channel condition within the ordered pair. In addition, comparing Fig. $3 \mathrm{~b}$ with Fig. 3a the aggregate throughput within each pair reaches a maximum value when the users in the pair reach the same downlink throughput and remains constant for higher values of $\beta_{P}$. In view of this result, for each ordered pair of users we select the value of $\beta_{P}$ which guarantees the same downlink performances of the users in a pair. This choice is motivated by the fact that at that point we have the same performances but with minimum aggregated power per pair 


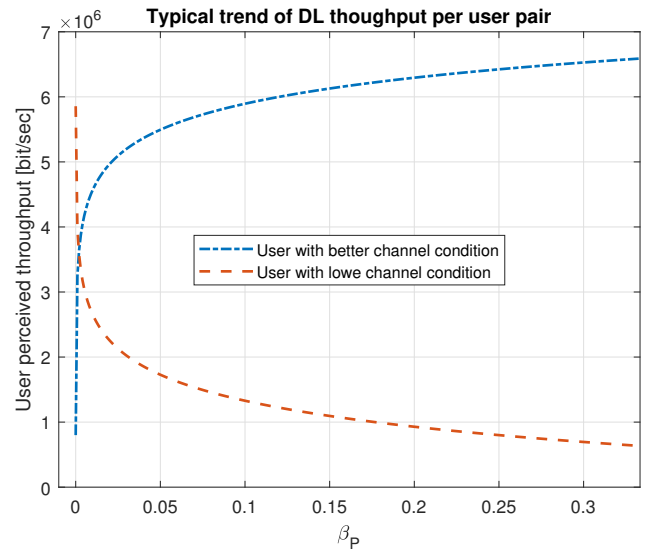

(a) Downlink perceived througut per user.

Fig. 3. Typical trend of downlink throughput along each ordered pair.

employed, i.e., higher local EE. The full proposed procedure for power optimization is summarized in Algorithm 3.

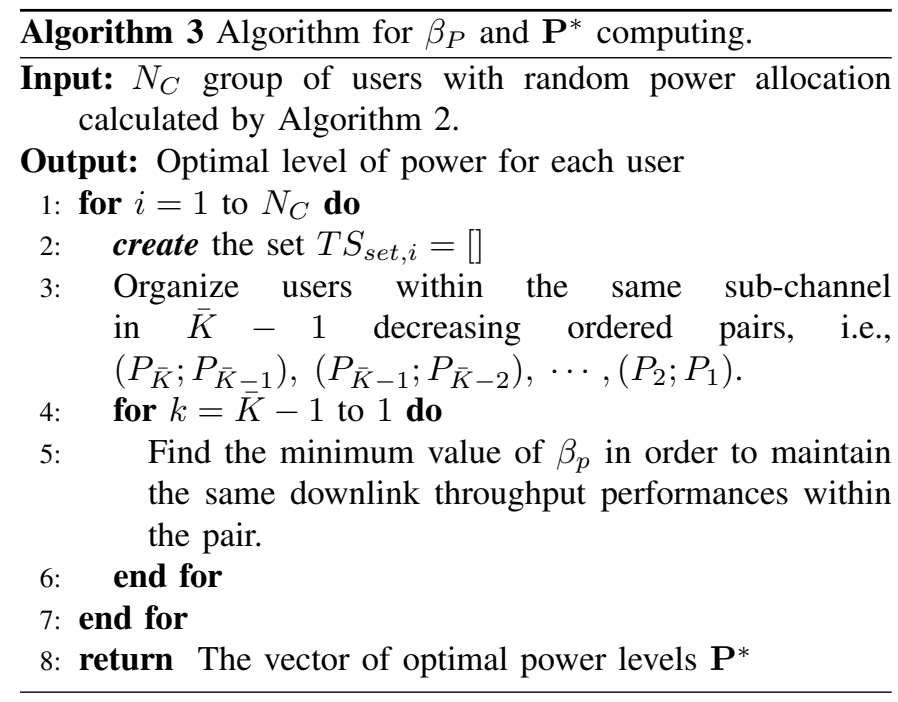

\section{B. Time resource allocation}

The next step of the optimization process is to find the optimal value of $\alpha$. However, calculating the first order derivative with respect to $\alpha$ of the objective function in (11), one can notice how this variable does not impact on the maximum value of the EE. In contrast, the value of $\alpha$ impacts the fairness among users. From this perspective, using the Jain's fairness definition [41], we formulate the following optimization problem for the downlink fairness maximization:

$$
\begin{aligned}
& \max _{\alpha} \frac{\left(\sum_{k=1}^{\bar{K}_{1}} R_{i, k, 1}+\sum_{k=1}^{\bar{K}_{2}} R_{i, k, 2}\right)^{2}}{K_{t o t, i} \times\left(\sum_{k=1}^{\bar{K}_{1}} R_{i, k, 1}^{2}+\sum_{k=1}^{\bar{K}_{2}} R_{i, k, 2}^{2}\right)} \\
& \text { s.t. } \quad 0<\alpha<1 \quad \forall k=1 \cdots K_{t o t, i},
\end{aligned}
$$

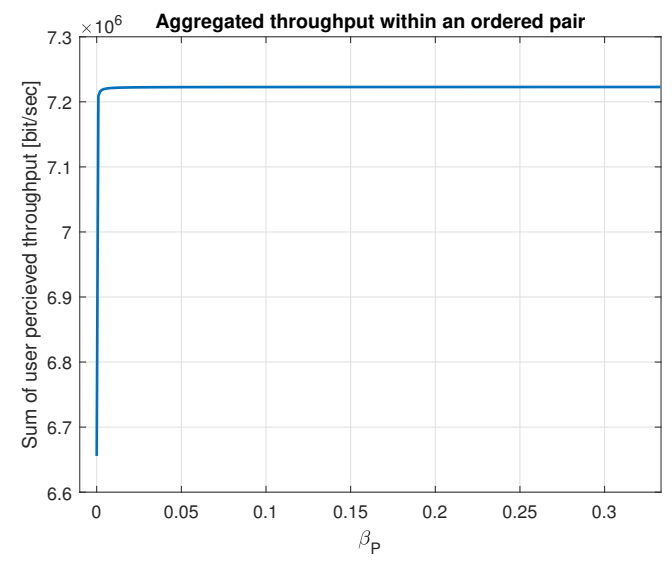

(b) Aggregated throughput.

where $\bar{K}_{1} \triangleq K_{i, 1}+1$ and $\bar{K}_{2} \triangleq K_{i, 2}+1$. After some mathematical manipulations, the objective function (23a) can be reformulated as:

$$
\frac{(\alpha z+w)^{2}}{K_{t o t, i} \times\left[\alpha^{2} x+(1-2 \alpha) y\right]},
$$

where

$$
\begin{aligned}
& x=\sum_{k=1}^{K_{\text {tot }, i}} R_{i, k}^{2} ; \quad y=\sum_{k=\bar{K}_{1}+1}^{K_{\text {tot }, i}} R_{i, k}^{2} ; \\
& z=\sum_{k=1}^{\bar{K}_{1}} R_{i, k}-\sum_{k=\bar{K}_{1}+1}^{K_{\text {tot }, i}} R_{i, k} ; \quad w=\sum_{k=\bar{K}_{1}+1}^{K_{\text {tot }, i}} R_{i, k} .
\end{aligned}
$$

For better comprehension, $R_{i, k}$ represents the following quantities:

$$
R_{i, k}= \begin{cases}\frac{R_{i, k, 1}}{\alpha} & 1 \leq k \leq \bar{K}_{1} ; \\ \frac{R_{i, k, 1}}{1-\alpha} & \bar{K}_{1}+1 \leq k \leq K_{t o t, i} .\end{cases}
$$

Then, calculating the first-order derivative with respect to $\alpha$ of (24) we obtain:

$$
-\frac{2(\alpha z+w)(\alpha(z y+w x)-(z y+w y))}{K_{t o t, i} \times\left[\alpha^{2} x+(1-2 \alpha) y\right]^{2}} .
$$

Albeit (27) admits two different solutions, we will choose the one which satisfies the constraint (23b). Then the closed-form expression for $\alpha_{\text {opt }}$ is:

$$
\alpha_{o p t}=\frac{y(z+w)}{(z y+w x)} .
$$

\section{Numerical Results}

In this section, we present simulation results obtained by applying the optimization procedures illustrated in Section III. As mentioned in Section II, we considered a simulation scenario where an UAV located at altitude $H$ provides coverage through TS-NOMA transmissions to a set of $N$ users, randomly distributed into a circular area of radius $R$ according to a PPP process. The noise power at the receivers in the 


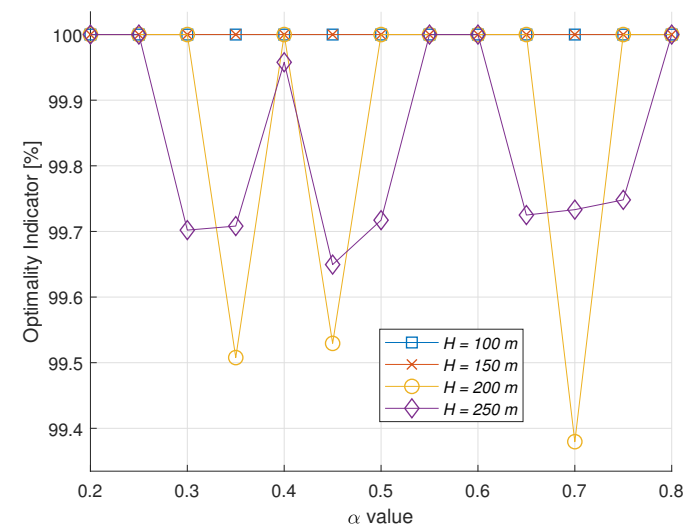

(a) $N=6 ; M=2$

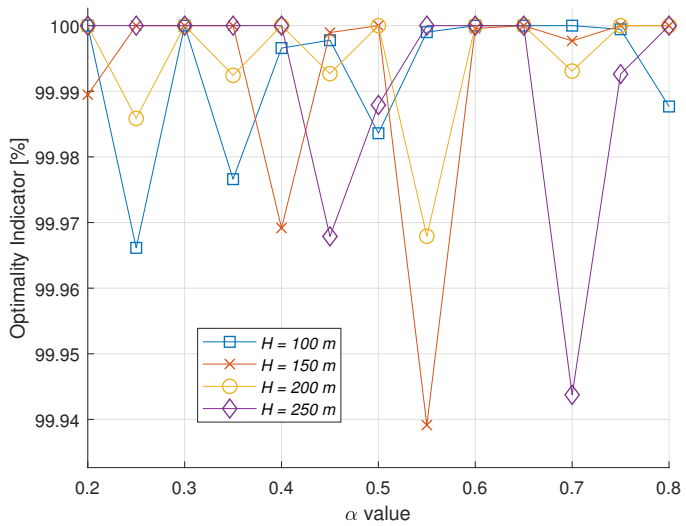

(c) $N=12 ; M=4$

Fig. 4. Optimality index variation over different network configurations.

whole bandwidth is $N_{0}=290 \cdot k_{B} \cdot B \cdot N F$, where $k_{B}$ and $N F$ are Boltzmann constant and noise figure at $9 \mathrm{~dB}$, respectively. Then, the noise power in each sub-channel is $N_{0} / N_{C}$. Herein, the simulation parameters used to investigate this scenario follow the study in [35] and are summarized in Table II. The performance of the proposed joint time

TABLE II

SIMULATION PARAMETERS.

\begin{tabular}{|l|r|}
\hline Parameter & Value \\
\hline$N$ (average number of nodes) & 150 \\
$H[\mathrm{~m}]$ & {$[100,150,200,250]$} \\
$\alpha$ & {$[0.2,0.3,0.4,0.6,0.7,0.8]$} \\
Bandwidth (MHz) & 40 \\
$N F(\mathrm{~dB})$ & 9 \\
$P_{\text {circ }}(\mathrm{mW})$ & 160 \\
$P_{\max }(\mathrm{mW})$ & 150 \\
Drain efficiency $\zeta$ & 1 \\
$a$ & 0.388 \\
$b$ & 11.95 \\
Cell radius [m] & 0.136 \\
Excessive attenuation factor $\gamma[\mathrm{dB}]$ & 500 \\
Pathloss exponent $\beta$ & 20 \\
$\sigma_{L O S}[\mathrm{~dB}]$ & 4 \\
$\sigma_{N L O S}[\mathrm{~dB}]$ & 4 \\
\end{tabular}

and power allocation scheme (JTPA) framework, in terms of

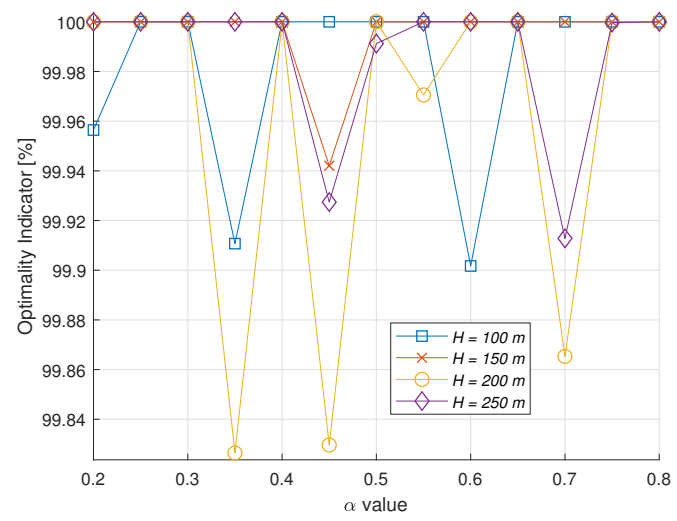

(b) $N=9 ; M=3$

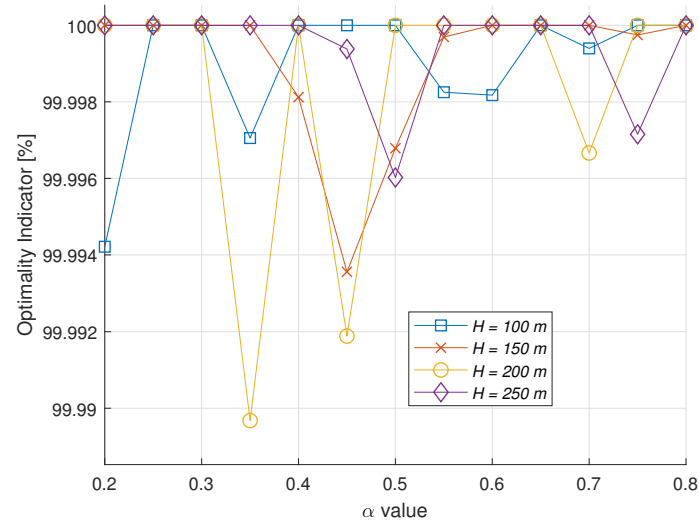

(d) $N=15 ; M=5$

$\mathrm{EE}$ and fairness maximization, has been compared with the following power allocation policies policies: $i$ ) the fixed power allocation (FPA) policy, ii) the random power allocation (RPA) policy illustrated in Section III-A, iii) the adoption of only power optimization policy (PA), and $i v$ ) the power allocation scheme proposed in [25], which is referred to as SOMSA-DC. Although the first two represent easy policies to implement in order to maintain the QoS constraint of the users, they have been chosen in order to highlight that they do not use the available power efficiently. The third policy permits to highlight the impact of the time optimization process in terms of fairness after the EE maximization. Finally, the SOMSADC framework provides a complete performance comparison in terms of user clustering complexity and both $\mathrm{EE}$ and throughput fairness maximization. These analyses have been performed for different values of $H$ and different initial values of $\alpha$, which are reported in Table II. For clarity, this section is organized in two subsections. Subsection IV-A discusses the user clustering procedure in terms of computational cost and optimality. Finally, the performance of the proposed JTPA framework in terms of EE and downlink throughput fairness maximization is provided in subsection IV-B. 


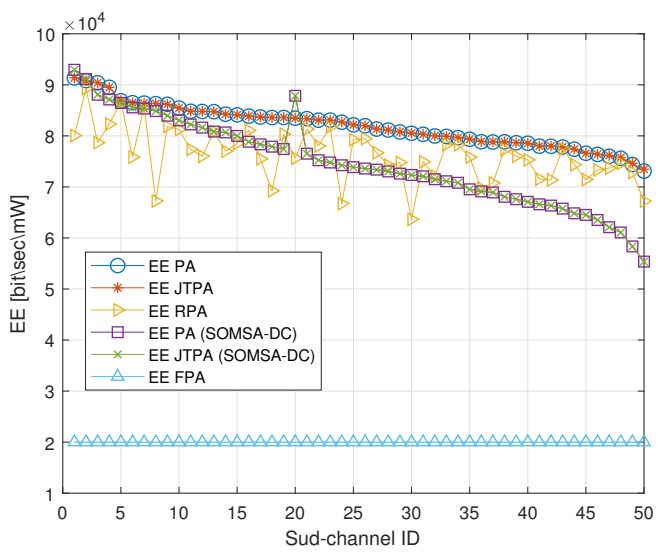

(a) $\alpha=0.2, H=200 \mathrm{~m}$.

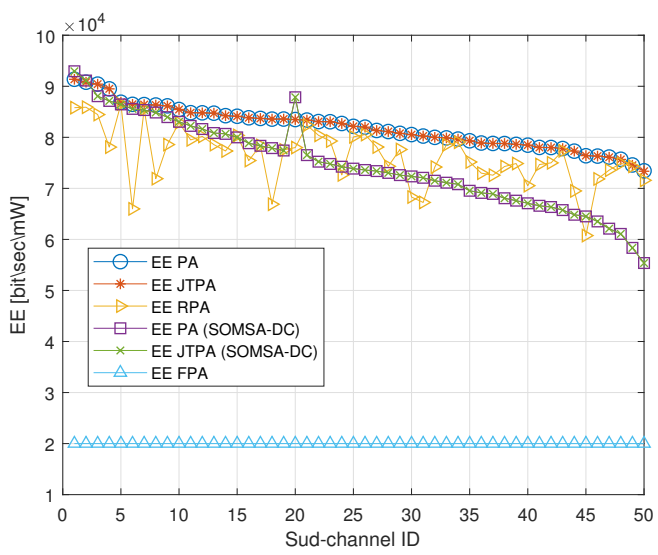

(c) $\alpha=0.6, H=200 \mathrm{~m}$.

Fig. 5. EE for different initial values of $\alpha$ and $H=200 \mathrm{~m}$.

\section{A. Computational cost and optimality}

In this subsection we discuss the proposed clustering framework in terms of computational cost and optimality. In order to have a direct comparison with the framework proposed in [25] and without loss of generality, we suppose that each cellcentre user will be multiplexed with $2 K=2$ cell-edge users, i.e., $K=1$ cell-edge user per sub-time slot and then $K_{i, 1}=$ $K_{i, 2}=1$. Under these assumptions, supposing to have $N$ users and $M$ sub-channels, the time complexity of the exhaustive search is $O\left(\frac{M !}{(3 !)^{N}}\right)$. Then, the logarithm of the complexity is $O(\ln (M !)-N \ln (3 !))$. Regarding the SOMSA algorithm proposed in [25], its worst case complexity occurs when all the users have the same preference list. In this case, the SOMSA algorithm has to compare $\left(\frac{N}{M}\right)^{2}\left(\sum_{i=1}^{N}(N-i)\right)$ configurations. Then, the worst case time complexity can be approximated as $O\left(\left(\frac{N}{M}\right)^{2}\right)$, i.e., $O\left(2(\ln (N)-\ln (M))+\ln \left(\sum_{i=1}^{N}(N-i)\right)\right.$ in logarithmic form. As mentioned in Section II-A, we propose to use a k-means++ based algorithm. As illustrated in [32] , the k-means ++ algorithm has a computational complexity of $\ln (N \times C \times d)$, with $C$ as the number of clusters and $d$ as the dimension of data point. Since we consider that the users are clustered in two groups, i.e., cell-centre users and cell-edge users, and $d=1$, the computational complexity of

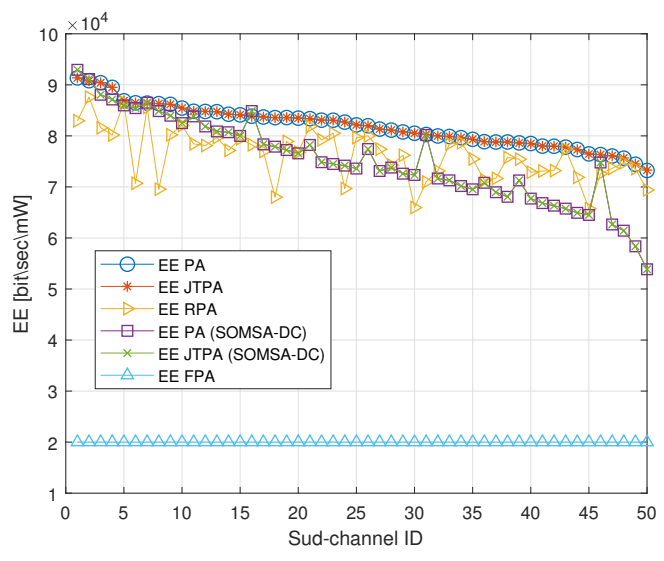

(b) $\alpha=0.4, H=200 \mathrm{~m}$.

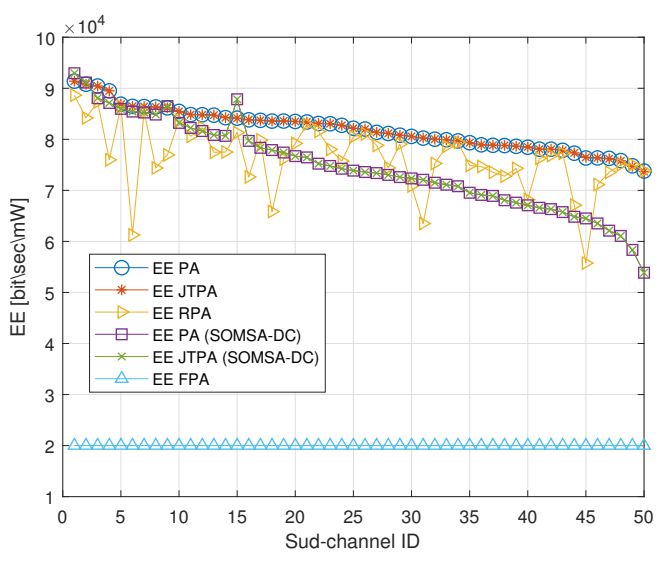

(d) $\alpha=0.8, H=200 \mathrm{~m}$.

our proposed clustering framework is $O(\ln (N))$, which is less than that of the SOMSA algorithm.

Regarding the discussion in terms of optimality, we compared the proposed clustering algorithm with the exhaustive search. In particular, indicating with $E E_{e x h}$ the maximum EE obtained by searching over all possible combinations of users and with $E E_{k m s}$ the maximum EE obtained using the $k$-means ++ based clustering algorithm, we used the following optimality index (OI) as performance indicator:

$$
O I=1-\frac{E E_{e x h}-E E_{k m s}}{E E_{e x h}} .
$$

Note that (29) indicates how close the EE achieved using the $k$-means ++ algorithm is to the one obtained by adopting the exhaustive search approach. The variation, in percentage, of the OI indicator for different values of $H$ and $\alpha$ is presented in Figs. (4a)-(4d). From these figures one can note that through the $k$-means ++ based algorithm is possible to reach levels of EE very close to the one obtained with exhaustive search approach..

As a result, the adoption of the proposed user-clustering scheme is more suitable for UAV-based scenario, since it can achieve EE performances close to optimal but with signifi- 


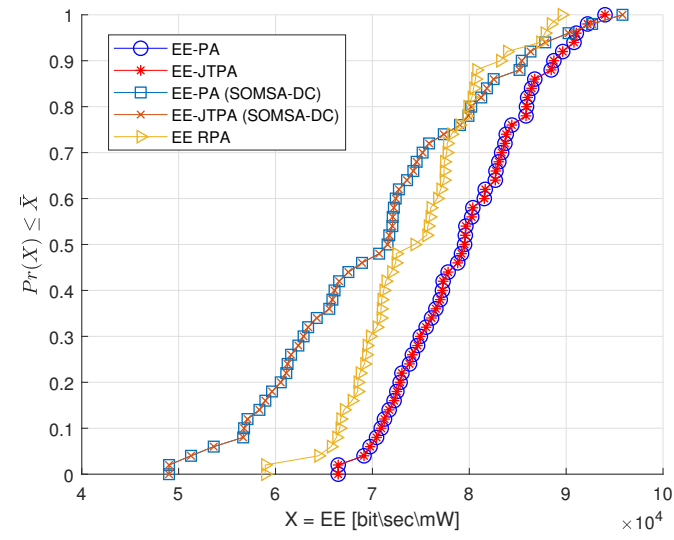

(a) $\alpha=0.5, H=100 \mathrm{~m}$.

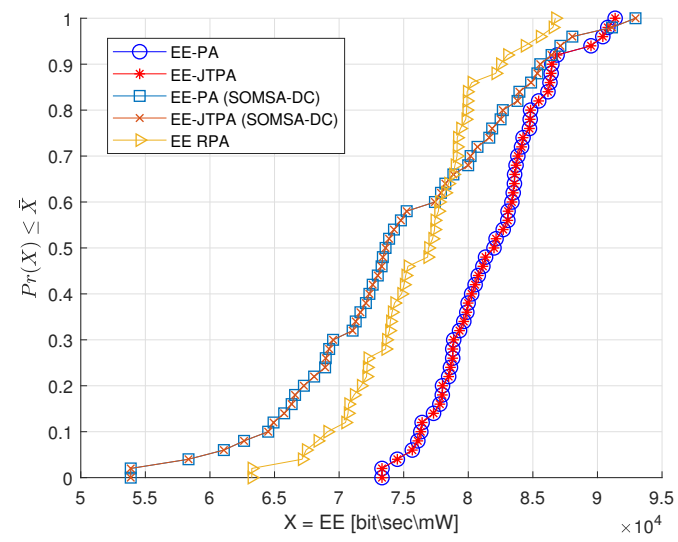

(c) $\alpha=0.5, H=200 \mathrm{~m}$.

Fig. 6. CDF of EE for different initial values of $H$ and $\alpha=0.5$.

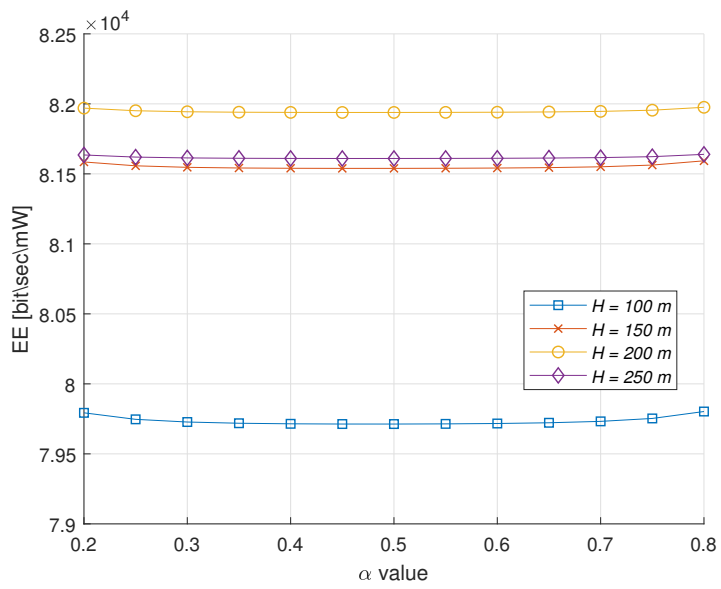

(a) Mean optimal EE of the proposed JTPA

Fig. 7. Mean optimal EE varying $\alpha$ and $H$. cantly reduced computational cost permitting then to prolong the UAV battery lifetime.

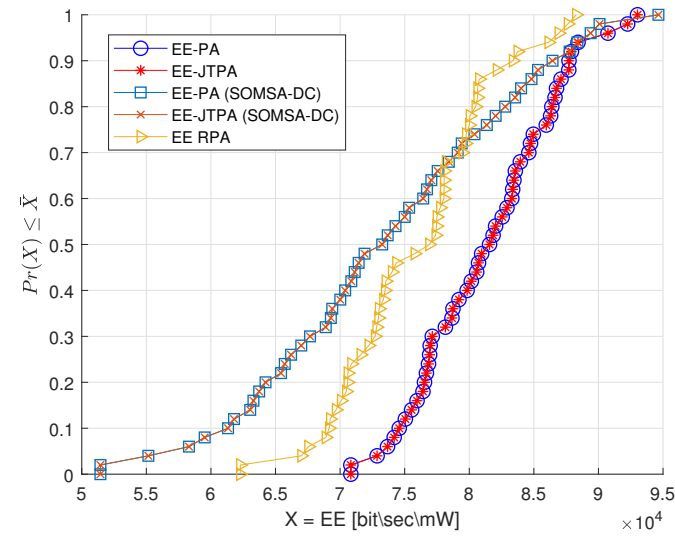

(b) $\alpha=0.5, H=150 \mathrm{~m}$.

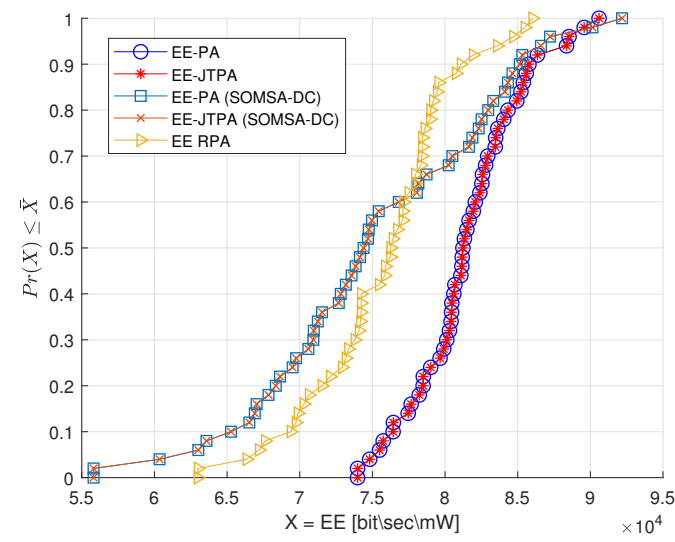

(d) $\alpha=0.5, H=250 \mathrm{~m}$.

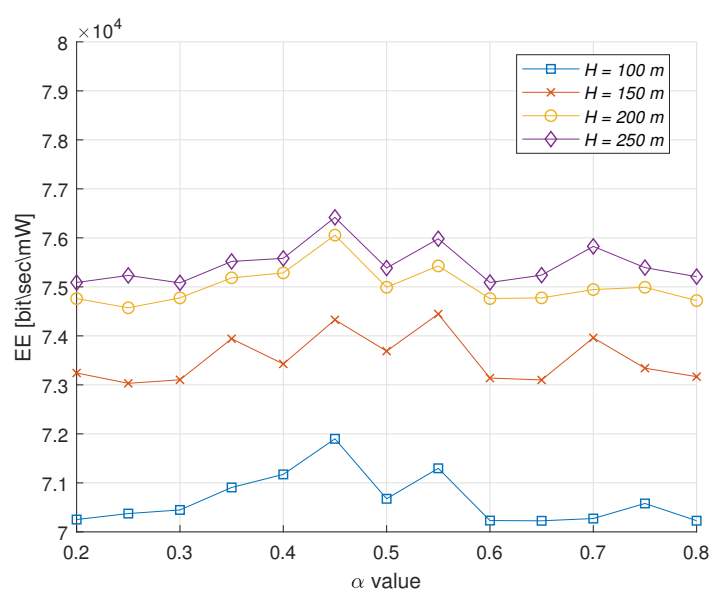

(b) Mean optimal EE of SOMSA-DC

\section{B. Network metrics maximization}

From Fig. 5, which presents the EE along each subchannel at a fixed UAV altitude $H$ and different values of $\alpha$, one can observe the following interesting results. Firstly, the 


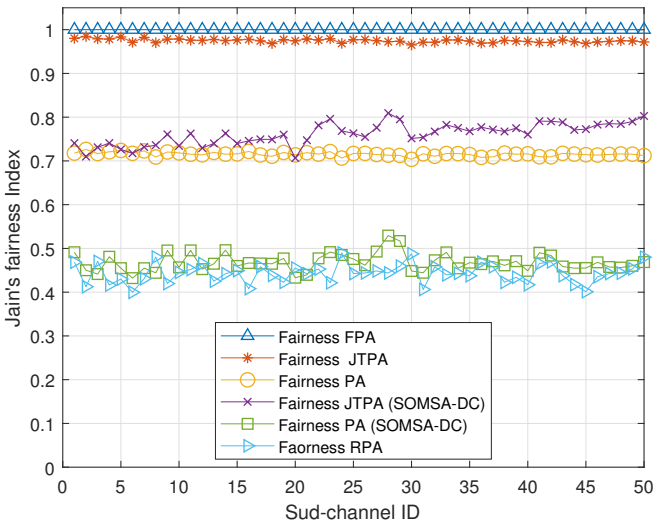

(a) $\alpha=0.2, H=200 \mathrm{~m}$.

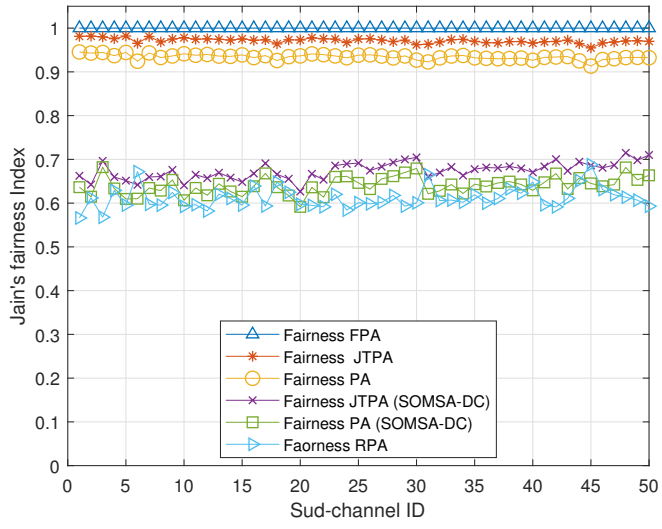

(c) $\alpha=0.6, H=200 \mathrm{~m}$.

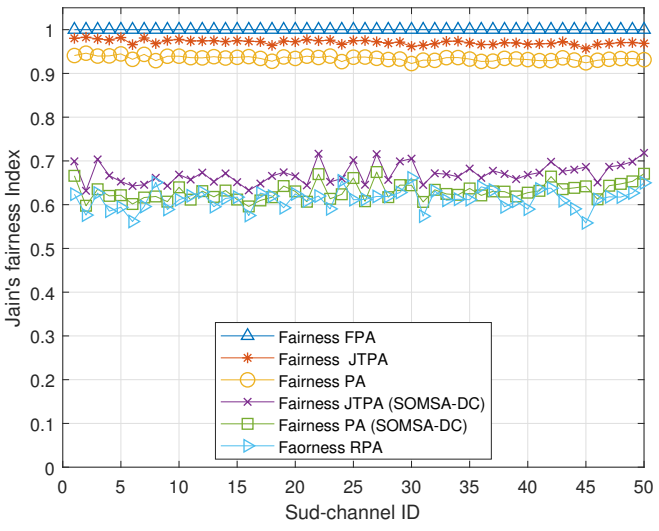

(b) $\alpha=0.4, H=200 \mathrm{~m}$.

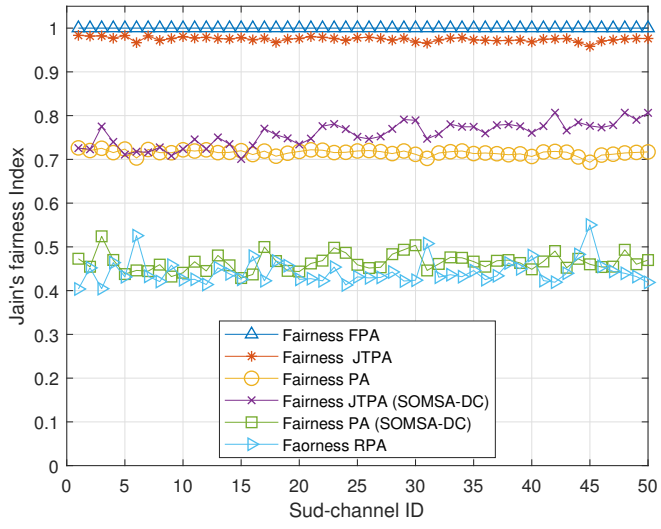

(d) $\alpha=0.8, H=200 \mathrm{~m}$.

Fig. 8. User fairness along each sub-channel for different initial values of $\alpha$ and $H=200 \mathrm{~m}$.

optimization procedure for power and time resource allocation provides better performances with respect to the FPA, RPA and SOMSA-DC allocation schemes, by allocating the proper amount of power which maximizes the EE along each subchannel. Secondly, as stated in Section III-B, the value of $\alpha$ does not impact on the EE maximization procedure. Indeed, it is possible to note how the full optimization policy reaches the same maximum points independently of the initial value of $\alpha$. Then, only the power allocation procedure is sufficient to reach the maximum of the EE. It is worthy to mention that the SOMSA-DC framework does not include a time optimization procedure; thus, for a fair comparison, we applied this optimization step to SOMSA-DC and refer to the new policy as JTPA (SOMSA-DC). In contrast, PA (SOMSA-DC) represents the legacy version of the SOMSA-DC framework. Although both legacy and the new version of SOMSA-DC reach the same final values, one can note how the initial value of $\alpha$ impacts the EE maximization, providing different trends of the EE per sub-channel by varying $\alpha$. Regarding the descending trend of the EE, it is related to the clustering policy. Indeed, observing Algorithm 1, as the sub-channel ID increases the channel gains of the users along that sub-channel decreases, i.e., more power is necessary to guarantee the QoS constraints. Then, since $P_{s u b}$ is supposed to be equal for each sub-channel, as a consequence the EE decreases with the sub-channel ID. Then, this confirms that the proposed framework finds the optimal values of power to maximize the EE respecting the power constraint.

In order to better highlight the gain of the proposed framework in terms of EE maximization, in Fig. 6 one can see the comparison between the cumulative distribution functions (CDF) of the EE obtained adopting different resource allocation methods.

Once again these figures confirm that: i) the proposed framework yields an improved performance when compared with RPA and SOMSA-DC frameworks in terms of EE; ii) the time allocation procedure does not impact the system in terms of the EE performance. For completeness, Fig. 7 shows how the EE varies for different values of UAV altitude $H$ and different initial values of $\alpha$. As expected, for a fixed value of $H$, the results in terms of EE of JTPA policy are independent from the initial value of the time slot duration (Fig. 7a ). In contrast, as mentioned before, the initial value of $\alpha$ impacts the EE reached by the SOMSA-DC framework (Fig. $7 b$ ). However, as also observed in Fig. 6, in both cases the EE varies with the UAV height. This behaviour can be explained 


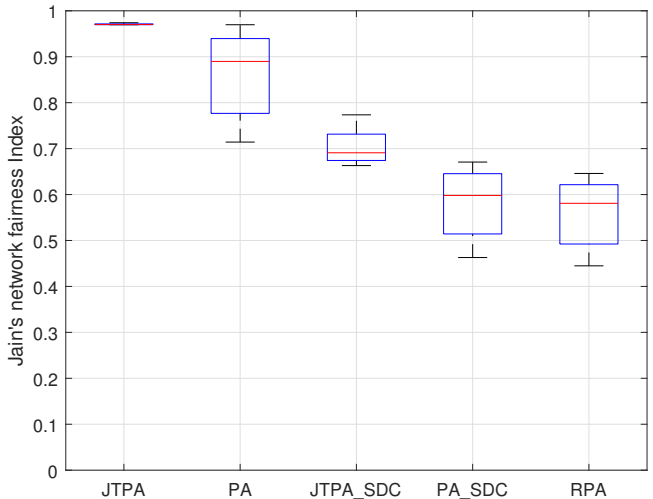

(a) $H=100 \mathrm{~m}$.

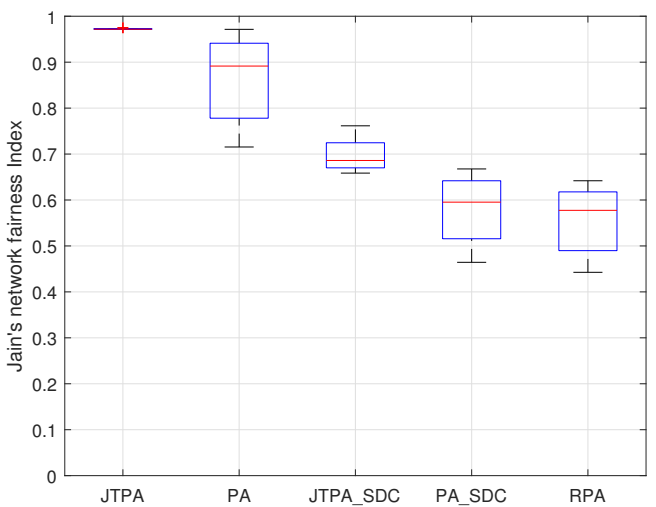

(c) $H=200 \mathrm{~m}$.

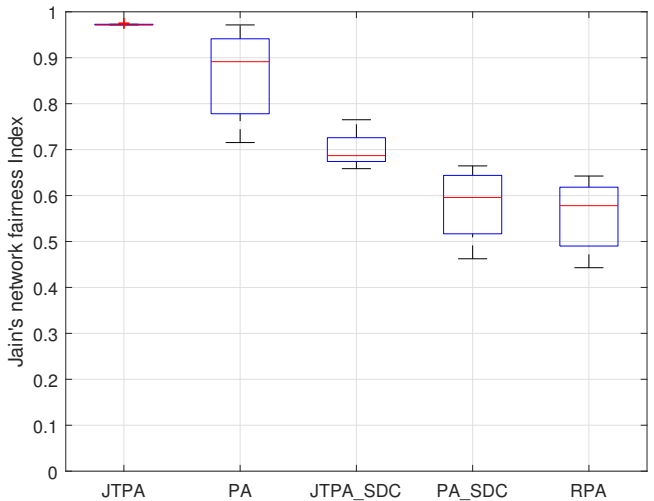

(b) $H=150 \mathrm{~m}$.

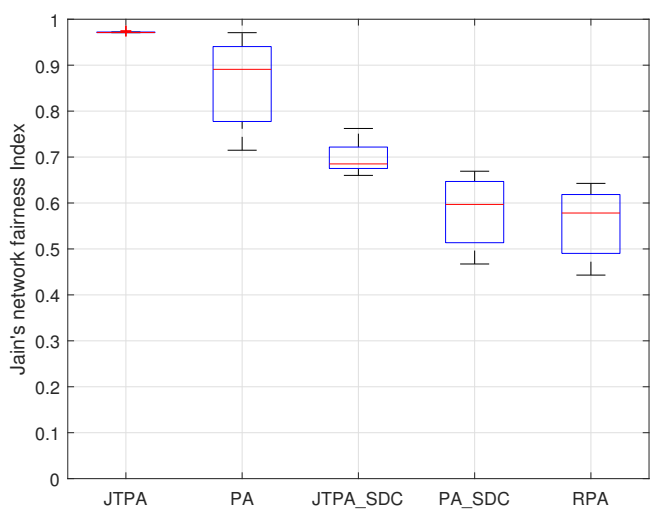

(d) $H=250 \mathrm{~m}$.

Fig. 9. Network fairness for different values of $H$ and power allocation policy.

by considering the UAV channel model. In particular, from (4) one can observe how an increase of $H$ corresponds to an increase of the elevation angle and then, according to (3), a further increase of $P_{r L O S}$. This means that more users will experience higher channel conditions and then higher downlink throughput with less power requirements, i.e., $\mathrm{EE}$ increases. However, after a certain altitude level, even if the $P_{r L O S}$ is high, the path loss component of (2) starts to be more dominant causing a channel gain degradation, i.e., the EE starts to decrease.

Fig. 8 illustrates the fairness index along each sub-channel obtained by adopting each specific policy for a fixed value of UAV altitude and different initial values of $\alpha$. One can note that the FPA policy reaches the maximum level of fairness and does not depend on the initial value of $\alpha$. This is because the FPA policy allocates the minimum level of power to each user, which permits to guarantee the QoS requirements. Since these constraints are supposed to be equal, ideally all users experience the same rate, i.e., maximum fairness. Furthermore, even if a higher level of fairness can be reached with a joint power and time resource allocation, the achievable fairness level of the SOMSA-DC policy is always lower than that of the proposed framework, both for the PA and JTPA cases. Finally, even from these figures, one can notice how the maximum value of fairness reached by the PA and PA (SOMSA-DC) policies depends on the initial value of $\alpha$. Indeed, for low $(0.2)$ or high (0.8) initial values of $\alpha$, through both PA and PA (SOMSA-DC) policies is not possible to reach a level of fairness higher than 0.7 and 0.5 , respectively. On the other hand, for initial values of $\alpha$ close to 0.5 , interestingly, the system performance of both PA and JTPA policies reaches the maximum fairness. This means that the procedure illustrated in Algorithm 3 for finding the value of $\beta_{P}$ implicitly maximizes the fairness by maintaining the maximum of $\mathrm{EE}$ when the time resources are equally divided. In other words, the proposed framework is able to jointly reach the maximum of both EE and throughput fairness only through the power allocation procedure.

For completeness, the box-plots provided in Figs. (9a)-(9d) are used to highlight better the dependence of the network fairness, achieved by each policy, on the UAV altitude and the initial value of $\alpha$. One can notice that the achievable fairness does not depend on the altitude, regardless of the policy. In addition, for a certain value of the UAV altitude, the interquartile range is zero only if the JTPA policy is adopted. This means that, in contrast to the other power allocation 
policies, the initial value of $\alpha$ does not influence the proposed framework in finding the optimal values of both power and time resources that jointly maximize the $\mathrm{EE}$ and the user fairness of the served area.

\section{Conclusions and Future Direction}

In this paper, we have introduced the concept of TSNOMA multiplexing in the context of UAV-enabled communications. Further, for the UAV-TS-NOMA communication system, we have proposed a user clustering procedure and formulated an optimization problem which, considering the QoS requirements of users and the power availability at the UAV as system constraints, aims to jointly maximize the EE and downlink throughput fairness in the network. Regarding the clustering procedure, we have illustrated how adopting a $k$-means ++ based solution permits to reach similar results as the exhaustive search, yet with a significantly lower computational cost, allowing then to reduce the processing energy at the UAV side. On the other hand, due to the nonlinearity of the optimization problem, we have proposed a dual-layer iterative algorithm in which $i$ ) the power resources are optimized and provided in a semi-closed form by using the Dinkelbach method in conjuction with some mathematical transformations, and subsequently ii) a closed-form expression for the optimal time resource allocation is obtained as the first-order derivative of the optimization function. By varying the main parameters that influence the optimization algorithm, we have evaluated the performance of the proposed algorithm through simulations. In particular, we have illustrated how in contrast to the constant power allocation, random power allocation, and both versions of the SOMSA-DC scheme proposed in [25], the proposed framework is able to efficiently use power and time resources, reaching the maximum of both EE and downlink network fairness while satisfying the optimization constraints. In addition, we have highlighted how the proposed power resource allocation procedure maximizes the fairness implicitly under particular conditions. Hence, the proposed UAV-TS-NOMA communication framework can find applicability in the context of disaster communications, where it can be adopted as a potential solution to perform energyefficient transmissions by saving energy at both transmitter and receiver.. However, since the performance of an UAV-based network is strongly dependent on the UAV trajectory path and position, as illustrated in [11], the authors believe that future directions of this work can be identified in the designed of an intelligent UAV-TS-NOMA network where multiple UAVs cooperate to optimize their transmission resources and trajectory path in order to maximize $\mathrm{EE}$ and throughput fairness in the context of disaster communication networks.

\section{REFERENCES}

[1] Cisco, "Cisco Visual Networking Index: Global Mobile Data Traffic Forecast Update, 20162021 White Paper." [Online]. Available: https://www.cisco.com/c/en/us/solutions/collateral/service-provider/ visual-networking-index-vni/mobile-white-paper-c11-520862.html

[2] Y. Saito, Y. Kishiyama, A. Benjebbour, T. Nakamura, A. Li, and K. Higuchi, "Non-Orthogonal Multiple Access (NOMA) for Cellular Future Radio Access," in Proc. IEEE 77th Vehicular Technology Conf. (VTC Spring), June 2013, pp. 1-5.
[3] S. M. R. Islam, N. Avazov, O. A. Dobre, and K. Kwak, "PowerDomain Non-Orthogonal Multiple Access (NOMA) in 5G Systems: Potentials and Challenges," IEEE Communications Surveys Tutorials, vol. 19, no. 2, pp. 721-742, May 2017.

[4] L. Dai, B. Wang, Y. Yuan, S. Han, C. I, and Z. Wang, "Non-Orthogonal Multiple Access for 5G: Solutions, Challenges, Opportunities, and Future Research Trends," IEEE Communications Magazine, vol. 53, no. 9, pp. 74-81, September 2015.

[5] S. Vanka, S. Srinivasa, Z. Gong, P. Vizi, K. Stamatiou, and M. Haenggi, "Superposition Coding Strategies: Design and Experimental Evaluation," IEEE Transactions on Wireless Communications, vol. 11, no. 7, pp. 2628-2639, Jul. 2012.

[6] Y. Zeng, R. Zhang, and T. J. Lim, "Wireless Communications with Unmanned Aerial Vehicles: Opportunities and Challenges," IEEE Communications Magazine, vol. 54, no. 5, pp. 36-42, May 2016.

[7] M. Mozaffari, W. Saad, M. Bennis, and M. Debbah, "Unmanned Aerial Vehicle With Underlaid Device-to-Device Communications: Performance and Tradeoffs," IEEE Transactions on Wireless Communications, vol. 15, no. 6, pp. 3949-3963, June 2016.

[8] B. Li, Z. Fei, and Y. Zhang, "UAV Communications for 5G and Beyond: Recent Advances and Future Trends," IEEE Internet of Things Journal, vol. 6, no. 2, pp. 2241-2263, April 2019.

[9] M. Erdelj, M. Krl, and E. Natalizio, "Wireless Sensor Networks and Multi-UAV Systems for Natural Disaster Management," Comput. Netw., vol. 124, no. C, p. 7286, Sep. 2017. [Online]. Available: https://doi.org/10.1016/j.comnet.2017.05.021

[10] J. Snchez-Garca, D. Reina, and S. Toral, "A Distributed PSO-based Exploration Algorithm for a UAV Network Assisting a Disaster Scenario," Future Generation Computer Systems, vol. 90, pp. $129-$ 148, August 2019. [Online]. Available: http://www.sciencedirect.com/ science/article/pii/S0167739X18303649

[11] T. Q. Duong, L. D. Nguyen, H. D. Tuan, and L. Hanzo, "Learning-Aided Realtime Performance Optimisation of Cognitive UAV-Assisted Disaster Communication," in Proc. IEEE Global Communications Conference, GLOBECOM 2019, Waikoloa, HI, USA, December 9-13,, 2019, pp. 16.

[12] L. Dai, B. Wang, Z. Ding, Z. Wang, S. Chen, and L. Hanzo, "A Survey of Non-Orthogonal Multiple Access for 5G," IEEE Communications Surveys Tutorials, vol. 20, no. 3, pp. 2294-2323, May 2018.

[13] M. F. Sohail and C. Y. Leow, "Maximized Fairness for NOMA Based Drone Communication System," in Proc. IEEE 13th Malaysia International Conference on Communications (MICC), 28-30 November. 2017, pp. 119-123.

[14] A. A. Nasir, H. D. Tuan, T. Q. Duong, and H. V. Poor, "UAV-Enabled Communication Using NOMA," IEEE Transactions on Communications, vol. 67, no. 7, pp. 5126-5138, July 2019.

[15] M. F. Sohail, C. Y. Leow, and S. Won, "Non-Orthogonal Multiple Access for Unmanned Aerial Vehicle Assisted Communication," IEEE Access, vol. 6, pp. 22716-22727, May 2018.

[16] M. M. Selim, M. Rihan, Y. Yang, L. Huang, Z. Quan, and J. Ma, "On the Outage Probability and Power Control of D2D Underlaying NOMA UAV-Assisted Networks," IEEE Access, vol. 7, pp. 1652516536, February 2019.

[17] J. Sun, Z. Wang, and Q. Huang, "Cyclical NOMA Based UAV-Enabled Wireless Network," IEEE Access, vol. 7, pp. 4248-4259, December 2018.

[18] X. Liu, J. Wang, N. Zhao, Y. Chen, S. Zhang, Z. Ding, and F. R. $\mathrm{Yu}$, "Placement and power allocation for noma-uav networks," IEEE Wireless Communications Letters, vol. 8, no. 3, pp. 965-968, March 2019.

[19] L. Gupta, R. Jain, and G. Vaszkun, "Survey of Important Issues in UAV Communication Networks," IEEE Communications Surveys Tutorials, vol. 18, no. 2, pp. 1123-1152, September 2016.

[20] M. Alzenad et al., "3-D Placement of an Unmanned Aerial Vehicle Base Station (UAV-BS) for Energy-Efficient Maximal Coverage," IEEE Wireless Communications Letters, vol. 6, no. 4, pp. 434-437, August 2017.

[21] L. Wang, B. Hu, and S. Chen, "Energy Efficient Placement of a Drone Base Station for Minimum Required Transmit Power," IEEE Wireless Communications Letters (Early Access), pp. 1-1, February 2018.

[22] M. Hua, Y. Wang, Z. Zhang, C. Li, Y. Huang, and L. Yang, "PowerEfficient Communication in UAV-Aided Wireless Sensor Networks," IEEE Communications Letters, vol. 22, no. 6, pp. 1264-1267, June 2018.

[23] M. Hua et al., "Energy-Efficient Cooperative Secure Transmission in Multi-UAV-Enabled Wireless Networks," IEEE Transactions on Vehicular Technology, vol. 68, no. 8, pp. 7761-7775, August 2019. 
[24] F. Fang, Z. Ding, W. Liang, and H. Zhang, "Optimal Energy Efficient Power Allocation With User fairness for Uplink MC-NOMA Systems," IEEE Wireless Communications Letters, vol. 8, no. 4, pp. 1133-1136, April 2019.

[25] F. Fang, H. Zhang, J. Cheng, and V. C. M. Leung, "Energy-Efficient Resource Allocation for Downlink Non-Orthogonal Multiple Access Network," IEEE Transactions on Communications, vol. 64, no. 9, pp 3722-3732, September 2016.

[26] Y. Li, H. Zhang, K. Long, S. Choi, and A. Nallanathan, "Resource Allocation for Optimizing Energy Efficiency in NOMA-based Fog UAV Wireless Networks," IEEE Network, vol. 34, no. 2, pp. 158-163, April 2020.

[27] M. F. Sohail, C. Y. Leow, and S. Won, "Energy-Efficient NonOrthogonal Multiple Access for UAV Communication System," IEEE Transactions on Vehicular Technology, vol. 68, no. 11, pp. 10834 10845, November 2019.

[28] M. B. Shahab, M. F. Kader, and S. Y. Shin, "A Virtual User Pairing Scheme to Optimally Utilize the Spectrum of Unpaired Users in NonOrthogonal Multiple Access," IEEE Signal Processing Letters, vol. 23, no. 12, pp. 1766-1770, December 2016.

[29] M. Hunukumbure, T. Moulsley, A. Oyawoye, S. Vadgama, and M. Wilson, "D2D for Energy Efficient Communications in Disaster and Emergency Situations," in Proc. 21st International Conference on Software, Telecommunications and Computer Networks-(SoftCOM 2013), 18-20 September 2013, pp. 1-5.

[30] A. Masaracchia, L. D. Nguyen, T. Q. Duong, and M.-N. Nguyen, "An Energy-Efficient Clustering and Routing Framework for Disaster Relief Network," IEEE Access, vol. 7, pp. 56 520-56 532, April 2019.

[31] M. B. Shahab and S. Y. Shin, "A Time Sharing Based Approach to Accommodate Similar Gain Users in NOMA for 5G Networks," in Proc. IEEE 42nd Conf. Local Computer Networks Workshops (LCN Workshops), October 2017, pp. 142-147.

[32] D. Arthur and S. Vassilvitskii, "k-means++: The Advantages of Careful Seeding," in Proceedings of the Eighteenth Annual ACM-SIAM Symposium on Discrete Algorithms, SODA 2007, New Orleans, Louisiana, USA, January 7-9, 2007, N. Bansal, K. Pruhs, and C. Stein, Eds. SIAM, 2007, pp. 1027-1035. [Online]. Available: http://dl.acm.org/citation.cfm?id=1283383.1283494

[33] Z. Ding, P. Fan, and H. V. Poor, "Impact of User Pairing on 5G Nonorthogonal Multiple-Access Downlink Transmissions," IEEE Transactions on Vehicular Technology, vol. 65, no. 8, pp. 6010-6023, August 2016.

[34] A. Masaracchia, D.-B. Ha, and N.-P. Le, "On the Optimal User Grouping in NOMA System Technology," EAI Endorsed Transactions on Industrial Networks and Intelligent Systems, vol. 6, no. 20, August 2019.

[35] A. Masaracchia, D. B. Da Costa, T. Q. Duong, M. Nguyen, and M. T. Nguyen, "A PSO-Based Approach for User-Pairing Schemes in NOMA Systems: Theory and Applications," IEEE Access, vol. 7, pp. 90550 90564 , July 2019.

[36] A. Al-Hourani, S. Kandeepan, and A. Jamalipour, "Modeling Air-toGround Path Loss for Low Altitude Platforms in Urban Environments," in Proc. IEEE Global Communications Conf, December 2014, pp. 28982904.

[37] A. Al-Hourani, S. Kandeepan, and S. Lardner, "Optimal LAP Altitude for Maximum Coverage," IEEE Wireless Communications Letters, vol. 3 , no. 6, pp. 569-572, December 2014.

[38] J. Holis and P. Pechac, "Elevation Dependent Shadowing Model for Mobile Communications via High Altitude Platforms in Built-Up Areas," IEEE Transactions on Antennas and Propagation, vol. 56, no. 4, pp. 1078-1084, April 2008.

[39] W. Dinkelbach, "On Nonlinear Fractional Programming," Manage. Sci., vol. 13, no. 7, pp. 492-498, March 1967. [Online]. Available: https://doi.org/10.1287/mnsc.13.7.492

[40] R. G. Ródenas, M. L. López, and D. Verastegui, "Extensions of Dinkelbach's Algorithm for Solving non-linear Fractional Programming Problems," Top, vol. 7, no. 1, pp. 33-70, June 1999. [Online]. Available: https://doi.org/10.1007/BF02564711

[41] R. K. Jain, D.-M. W. Chiu, and W. R. Hawe, "A Quantitative Measure of Fairness and Discrimination for Resource Allocation in Shared Computer System," Eastern Research Laboratory, Digital Equipment Corporation, Hudson, MA, 1984

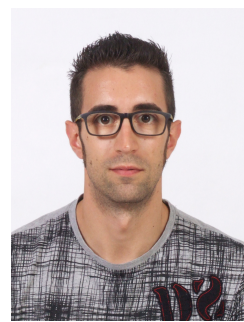

Antonino Masaracchia received the Ph.D. degree in electronics and telecommunications engineering from the University of Palermo, Italy, in 2016. His $\mathrm{Ph} . \mathrm{D}$. studies were conducted in joint supervision with the Institute of Informatics and Telematics (IIT), National Research Council (CNR), Pisa, Italy, and the obtained results have been important contributions from IIT to the FP7-MOTO European Project. Since 2018, he is Research Fellow with the Centre for Wireless Innovation, Queens University Belfast, U.K. His research interests include heterogeneous networks, convex optimization and machine learning techniques, wireless communication, and green communication networking. He currently serves as a Guest Editor for IET Communications and a special issue on Radio Frequency Energy Harvesting and Wireless Power Transfer published by MDPI Electronics for which serves also as Topic Editor. He serves as guest editor for a special issue on Controls, Communications and Networking for Ad-hoc Mobile Sensor Networks published by ICSES Transactions on Computer Networks and Communications. He served as guest editor for a special issue on Reliable Communication for Emerging Wireless Networks published by Mobile Networks and Applications (ACM/Springer).

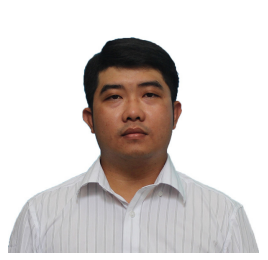

Long D. Nguyen was born in Dong Nai, Vietnam. He received his B.S. degree in Electrical and Electronics Engineering and M.S. degree in Telecommunication Engineering from Ho Chi Minh City University of Technology (HCMUT), Vietnam, in 2013 and 2015, respectively. He received his Ph.D. degree in Electronics and Electrical Engineering from Queen's Univerisity Belfast (QUB), UK, in 2018. He was a Research Fellow at Queen's University Belfast, UK for a part of Newton project (20182019). He is currently with Duy Tan University as an Adjunct Assistant Professor and Dong Nai University in Vietnam as an Assistant Professor. His research interests include convex optimization techniques for resource management in wireless communications, energy efficiency approaches, and real-time embedded optimization for wireless networks and Internet of Things (IoTs). He is currently serving as a reviewer for IEEE Trans on Wireless Communications, IEEE Trans on Communications, IEEE Access, IEEE Communication Letter, IET Communications and server international conferences. Dr. Nguyen was awarded the Best Paper Award at the IEEE Digital Signal Processing (DSP) 2017, the IEEE International Conference on Recent Advances in Signal Processing, Telecommunication and Computing (Sigtelcom) 2018, the IEEE International Conference on Communications (ICC) 2019, the International Wireless Communications \& Mobile Computing Conference (IWCMC) 2019 and the IEEE Global Communications Conference (GLOBECOM) 2019. He was also awarded the Exemplary Reviewer Award in IEEE Communications Letters 2018.

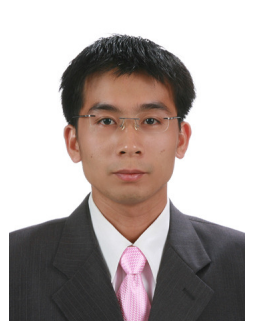

Trung Q. Duong ((S'05, M'12, SM'13) received his $\mathrm{Ph} . \mathrm{D}$. degree in Telecommunications Systems from Blekinge Institute of Technology (BTH), Sweden in the end of 2012. Currently, he is a Professor with Queen's University Belfast (UK), where he was a Lecturer (Assistant Professor) (2013-2017), a Reader (Associate Professor) (2018-2020), and Full Professor from August 2020. His current research interests include wireless communications, machine learning, realtime optimisation, big data, and IoT applications to disaster management, airquality monitoring, flood monitoring, smart agriculture, healthcare and smart cities. He is the author or co-author of over $350+$ technical papers published in scientific journals $(220+$ articles) and presented at international conferences (140+ papers). Dr. Duong currently serves as an Editor for the IEEE TRANSACTIONS ON WIRELESS COMMUNICATIONS, IEEE TRANSACTIONS ON COMMUNiCATIONS, and an Executive Editor for IEEE COMMUNICATIONS LetTers. He was awarded the Best Paper Award at the IEEE Vehicular Technology Conference (VTC-Spring) in 2013, IEEE International Conference on Communications (ICC) 2014, IEEE Global Communications Conference (GLOBECOM) 2016 and 2019, IEEE Digital Signal Processing Conference (DSP) 2017, and International Wireless Communications \& Mobile Computing Conference (IWCMC) 2019. He is the recipient of prestigious Royal Academy of Engineering Research Fellowship (2016-2020) and has won a prestigious Newton Prize 2017. 


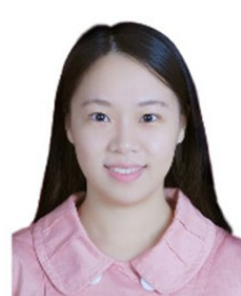

Cheng Yin received the B.Eng degree in Communication Engineering from Hangzhou Dianzi University, China, in 2016, and the PhD degree in Wireless Communication from Queens University Belfast, UK, in 2019. She is currently a Research Fellow at the Institute of Electronics, Communications and Information Technology of Queens University Belfast. Her research interests include big data analysis, machine learning, physical layer security, cognitive radio and energy harvesting communications.

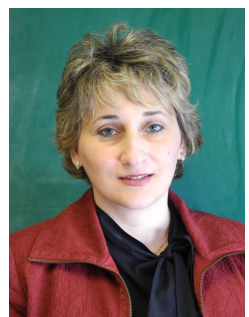

Octavia A. Dobre (M'05, SM'07, F'20) received the Dipl. Ing. and Ph.D. degrees from the Polytechnic Institute of Bucharest, Romania, in 1991 and 2000, respectively. Between 2002 and 2005, she was with New Jersey Institute of Technology, USA. In 2005, she joined Memorial University, Canada, where she is currently Professor and Research Chair. She was a Visiting Professor with Massachusetts Institute of Technology, USA and Universit de Bretagne Occidentale, France. Her research interests encompass various wireless technologies, such as non-orthogonal multiple access and full duplex, as well as optical and underwater communications. She has co-authored over 300 refereed journal papers in these areas. Dr. Dobre serves as the Editor-in-Chief (EiC) of the IEEE Open Journal of the Communications Society. She was the EiC of the IEEE Communications Letters, Senior Editor, Editor, and Guest Editor for various prestigious journals and magazines. She also served as General Chair, Technical Program Co-Chair, Tutorial Co-Chair, and Technical CoChair of symposia at numerous conferences. Dr. Dobre was a Royal Society Scholar and a Fulbright Scholar. She obtained Best Paper Awards at various conferences, including IEEE ICC, IEEE Globecom, and IEEE WCNC. Dr. Dobre is a Distinguished Lecturer of the IEEE Communications Society and a Fellow of the Engineering Institute of Canada.

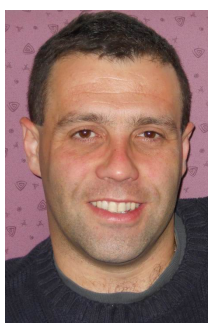

Emiliano Garcia-Palacios is a Senior Lecturer (Associate Professor) at Queens University Belfast, UK. He received his $\mathrm{PhD}$ from Queens University Belfast in 2000. His research interests include $5 \mathrm{G}$, wireless protocols, security, wireless resource allocation and optimization, machine learning, next generation gigabit networks, sensor networks, energy harvesting and low power IoT communications. He has supervised several $\mathrm{PhDs}$ in the aforementioned areas and has over 80 publications in international journals and international conferences. His latest research interest is in applying low cost, low energy IoT solutions to global challenges. 